\title{
molecules
}

ISSN 1420-3049

www.mdpi.com/journal/molecules

Article

\section{Synthesis and Electrophilic Substitutions of Novel Pyrazolo[1,5-c]-1,2,4-triazolo[4,3-a]pyrimidines}

\section{Kamal F.M. Atta}

Chemistry Department, Faculty of Science, Alexandria University, Ibrahimia P.O. Box 426, Alexandria 21321, Egypt; E-Mail: prof.kf_atta@yahoo.com; Tel.: 002035917883; Fax: 002035932488

Received: 30 June 2011; in revised form: 1 August 2011 / Accepted: 4 August 2011 /

Published: 18 August 2011

\begin{abstract}
Aryl-7-hydrazino-2-phenylpyrazolo[1,5-c]pyrimidines $\mathbf{1}$ were used as precursors for the preparation of a new series of 5-aryl-8-phenylpyrazolo[1,5-c]-1,2,4triazolo[4,3-a]pyrimidines 2 . The reactions of 2 with certain electrophilic reagents gave the respective 6-substituted derivatives 3-5 rather than the 7-isomeric products. Formylation of the key compounds 1 with ethyl formate yielded the formyl derivatives 6. Furthermore, boiling of compounds 1 with acetic acid afforded 7-acetylhydrazino-5aryl-2-phenylpyrazolo[1,5-c]pyrimidines 7. Bromination of 7 yielded the dibromoderivatives 8, while their iodination and nitration gave the monosubstituted derivatives 9 and 10, respectively. Also, treatment of $\mathbf{1}$ with boiling acetic anhydride yielded the triacetyl derivatives 11. The structure of synthesized products was confirmed by elemental analyses, IR, ${ }^{1} \mathrm{H}$ NMR and MS spectra.
\end{abstract}

Keywords: pyrazolo[1,5-c]-1,2,4-triazolo[4,3-a]pyrimidines; synthesis; electrophilic substitution reactions; dehydrative cyclization

\section{Introduction}

The pyrazolo[1,5-c]pyrimidine ring represents a biologically and synthetically important class of compounds. Many pyrazolo[1,5-c]pyrimidines are known to possess significant hypnotic, tranquilizing, fungicidal, insecticidal and antibacterial activities [1-3]. Also, the coordination of pyrazolo[1,5-c]pyrimidines to transition metal ions such as $\mathrm{Cu}^{+2}$ and $\mathrm{Ni}^{+2}$ enhances their biological activities [4-7]. 
In the last few decades, the chemistry of 1,2,4-triazoles and their fused heterocyclic derivatives has received considerable attention owing to their synthetic and effective biological importance. 1,2,4Triazole moieties have been incorporated into a wide variety of therapeutically interesting drug candidates, e.g., triazolam [8], alprazolam [9], etizolam [10], and furacylin [11], including antiinflammatories, central nervous system stimulants, sedatives, anti-anxiety compounds, antimicrobial agents [12-15] and antimycotic ones such as fluconazole, intraconazole, voriconazole [16,17].

The above mentioned therapeutic activity has prompted the present investigation to synthesize the pyrazolo[1,5-c]-1,2,4-triazolo[4,3-a]pyrimidines ring system 2 and a new series of 7-acetylhydrazino5-aryl-2-phenylpyrazolo[1,5-c]pyrimidine derivatives 7 .

\section{Results and Discussion}

Much work from our laboratory has utilized hydrazino heterocycles as raw materials for the synthesis of various types of heterocyclic compounds [18-21]. In the present investigation, the target pyrazolotriazolopyrimidine compounds were synthesized from 5-aryl-7-hydrazino-2-phenylpyrazolo[1,5-c]pyrimidines 1a-d that were prepared via a sequence of reactions from ethyl phenylpropiolate [2,22]. Heating of 1a-d with formic acid under reflux yielded a novel series of 5-aryl8-phenylpyrazolo[1,5-c]-1,2,4-triazolo[4,3-a]pyrimidines 2a-d (Scheme 1). The structures of 2a-d were deduced from their spectral analyses. Thus, the ${ }^{1} \mathrm{H}-\mathrm{NMR}$ spectra revealed the presence of three singlets for the pyrazole ring proton at $\delta_{\mathrm{H}} 6.91-7.33 \mathrm{ppm}$, of the pyrimidine ring proton at $\delta_{\mathrm{H}}$ 7.43-7.44 ppm and of triazole ring proton at $\delta_{\mathrm{H}} 8.53-9.00 \mathrm{ppm}$, in addition to the aromatic ring protons and the absence of NH signals. The MS spectra also showed a molecular ion peak as a base peak that indicated the stability of this ring.

The electrophilic substitution reactions of pyrazolotriazolopyrimidines 2a-d such as bromination with bromine, iodination with iodine monochloride and nitration with nitric and sulfuric acids in glacial acetic acid gave the respective 5-aryl-6-bromo-, 5-aryl-6-iodo- and 5-aryl-6-nitro-8phenylpyrazolo[1,5-c]-1,2,4-triazolo[4,3-a]pyrimidines 3a-d, 4a-d and 5a-d. Their ${ }^{1} \mathrm{H}-\mathrm{NMR}$ spectra revealed the absence of signals due to the pyrimidine ring proton and the presence of a pyrazole ring proton signal at $\delta_{\mathrm{H}} 6.81-7.33 \mathrm{ppm}$ and a triazole ring proton singlet at $\delta_{\mathrm{H}} 8.52-9.04 \mathrm{ppm}$, together with the aromatic proton signals at $\delta_{\mathrm{H}} 7.12-8.14 \mathrm{ppm}$. The structure of these derivatives were also confirmed from their mass spectral data.

Treatment of 1a-d with boiling ethyl formate afforded 5-aryl-7-formylhydrazino-2phenylpyrazolo[1,5-c]pyrimidines 6a-d. Their ${ }^{1} \mathrm{H}-\mathrm{NMR}$ spectra showed a new characteristic signal at $\delta_{\mathrm{H}} 7.98-8.08 \mathrm{ppm}$ corresponding to the formyl proton, in addition to the aromatic ring protons at $\delta_{\mathrm{H}}$ 7.20-7.99 ppm, with other characteristic signals; a singlet for the exchangeable two NH protons which were assigned at $\delta_{\mathrm{H}} 4.73-4.80 \mathrm{ppm}$, a singlet at $\delta_{\mathrm{H}} 6.68-6.72 \mathrm{ppm}$ for the pyrazole ring proton and a singlet at $\delta_{\mathrm{H}} 7.19-7.29 \mathrm{ppm}$ for the pyrimidine ring proton. 
Scheme 1. Synthesis and electrophilic substitution reactions of pyrazolotriazolopyrimidines.

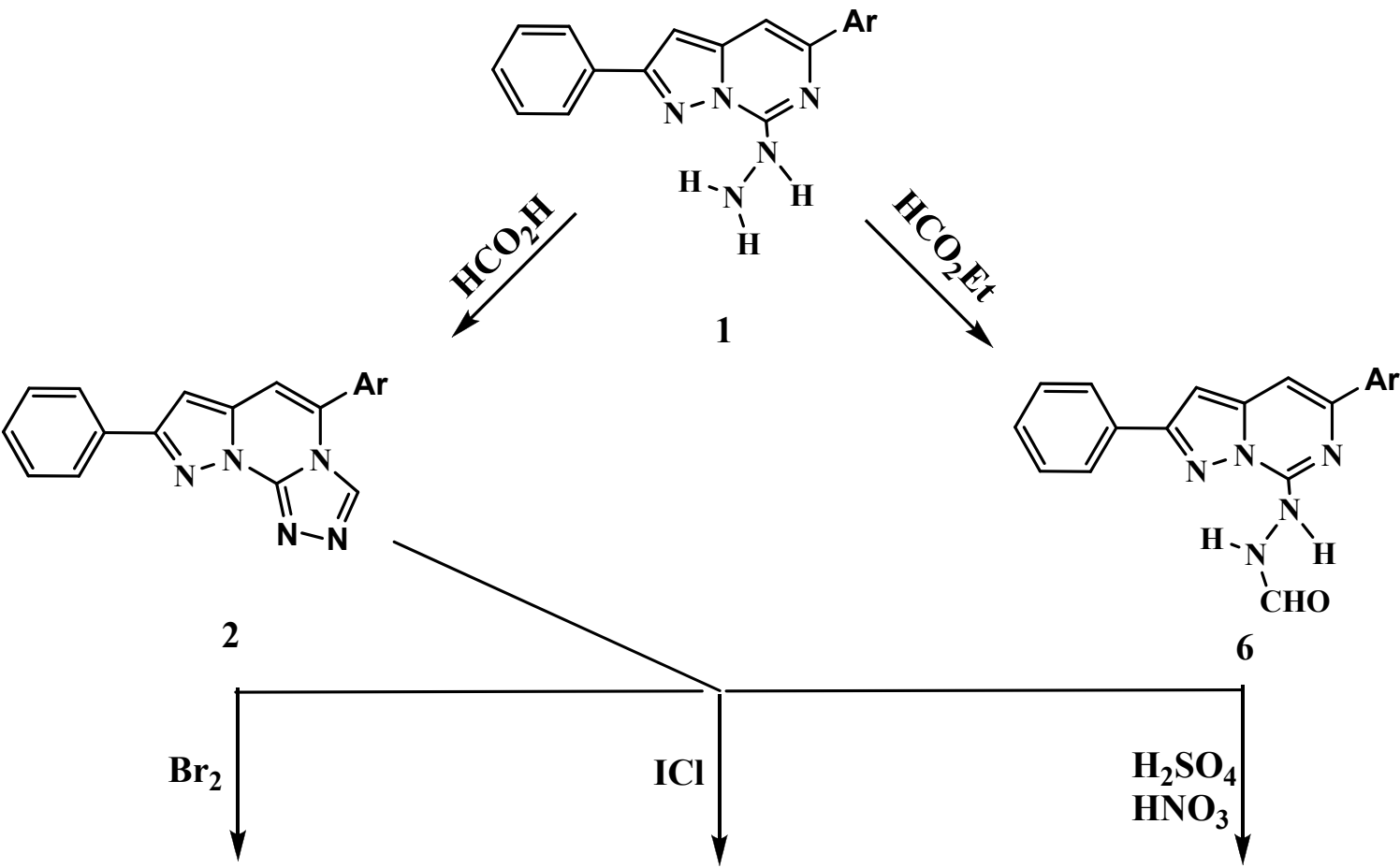

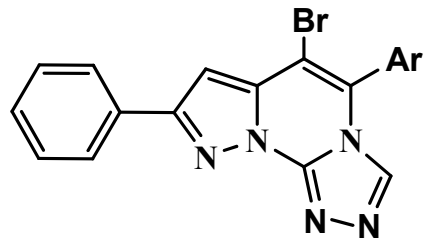

3

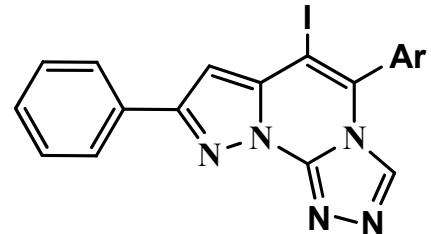

4<smiles></smiles>

5

$$
\mathrm{Ar}=\mathrm{C}_{6} \mathrm{H}_{5^{-}}(\mathrm{a}), \mathrm{p}-\mathrm{Me}-\mathrm{C}_{6} \mathrm{H}_{4^{-}}(\mathrm{b}), \mathrm{p}-\mathrm{MeO}-\mathrm{C}_{6} \mathrm{H}_{4^{-}}(\mathrm{c}), \mathrm{p}-\mathrm{Cl}-\mathrm{C}_{6} \mathrm{H}_{4^{-}}(\mathrm{d})
$$

Boiling of hydrazine derivatives 1a-d with acetic acid under reflux afforded 7-acetylhydrazino-5aryl-2-phenylpyrazolo[1,5-c]pyrimidines 7a-d (Scheme 2). The structures of 7a-d were confirmed by their ${ }^{1} \mathrm{H}-\mathrm{NMR}$ spectra, which revealed an acetyl group proton singlet at $\delta_{\mathrm{H}} 2.04-2.36 \mathrm{ppm}$, in addition to the characteristic signals of pyrazole and pyrimidine ring protons, two exchangeable $\mathrm{NH}$ protons and aromatic ring protons. The mass spectra of 7a-d which showed their molecular ion peaks as a base peak also confirmed the structures.

Next, the electrophilic substitution reaction of 7a-d via bromination with bromine in acetic acid gave the unexpected dibromo derivatives 8a-d rather than the monobromo derivatives. Their ${ }^{1} \mathrm{H}-\mathrm{NMR}$ spectra showed the absence of the signals of both pyrazole and pyrimidine ring protons and the presence of acetyl group protons, in addition to the other characteristic signals. These unexpected obtained products may be due to the excess bromine added to obtain a homogenous reaction mixture.

Iodination and nitration of 7a-d yielded the expected 5-aryl-2-phenyl-3-substituted-pyrazolo[1,5c]pyrimidine derivatives 9a-d and 10a-d, respectively. Their ${ }^{1} \mathrm{H}-\mathrm{NMR}$ spectra revealed the absence of pyrazole ring proton and the presence of pyrimidine ring proton at $\delta_{\mathrm{H}} 7.18-7.73 \mathrm{ppm}$ as well as the other characteristic signals. 
Scheme 2. Synthesis and electrophilic substitution reactions of 7-acetylhydrazinopyrazolopyrimidines.

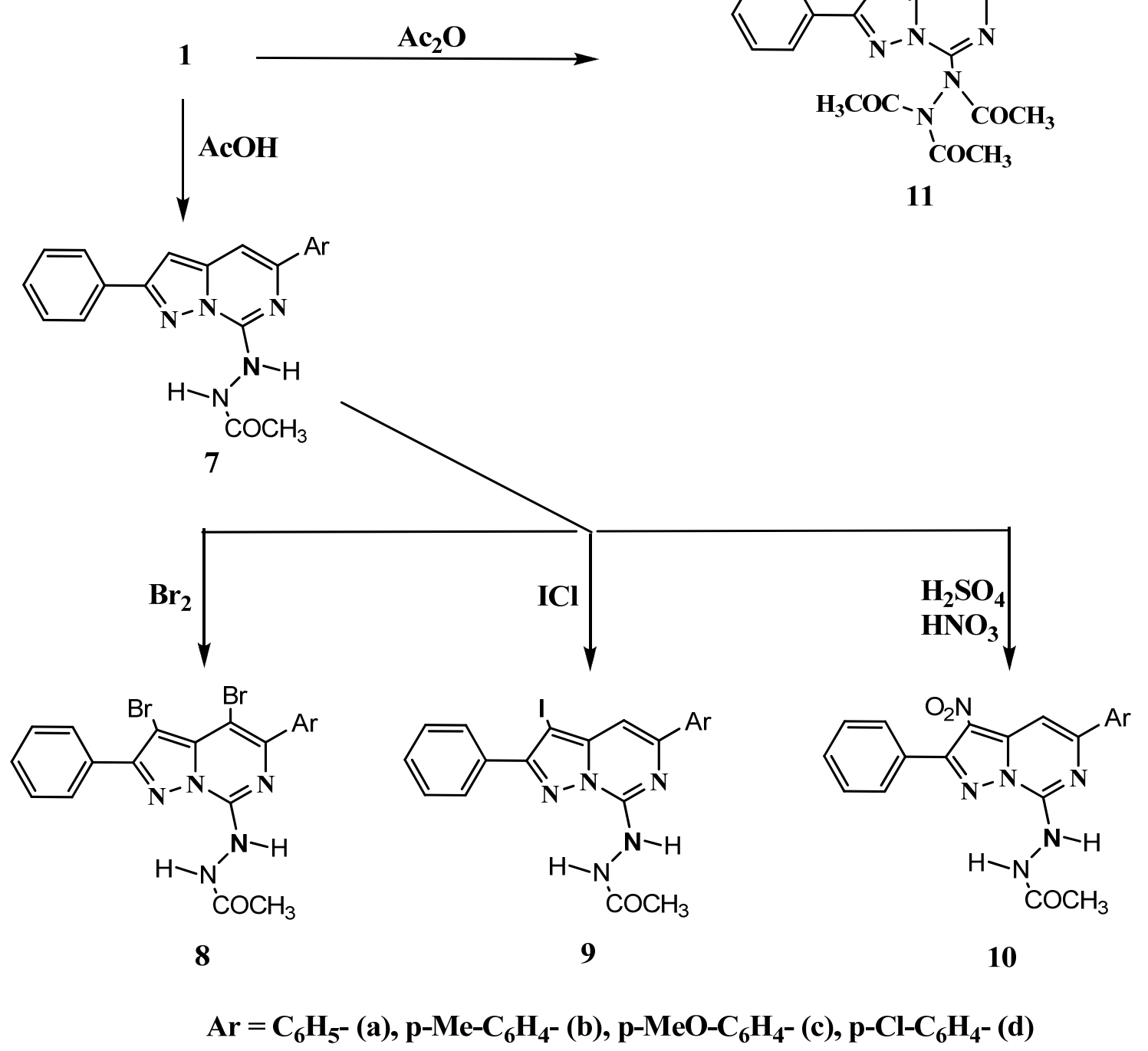

Furthermore, acetylation of 1a-d with boiling acetic anhydride afforded the triacetyl derivatives 11a-d. Their ${ }^{1} \mathrm{H}$-NMR spectra revealed the absence of the $\mathrm{NH}$ protons of the starting hydrazine derivatives and the presence of signals at $\delta_{\mathrm{H}} 2.42-2.56 \mathrm{ppm}$ corresponding to the three acetyl groups protons, as well as a singlet at $\delta_{\mathrm{H}} 6.87-7.28 \mathrm{ppm}$ for the pyrazole ring proton and a singlet at $\delta_{\mathrm{H}} 7.70-8.24 \mathrm{ppm}$ for the pyrimidine ring proton, in addition to the aromatic ring protons at $\delta_{\mathrm{H}} 6.98-8.05 \mathrm{ppm}$. The structures of 11a-d were also confirmed by their MS spectra which showed fragmentation process involving a sequential elimination of two ketene molecules to give the most stable one $\left(\mathrm{M}^{+}-2 \mathrm{CH}_{2} \mathrm{CO}\right)$ as a base peak.

\section{Experimental}

\subsection{General}

Melting points were determined on a Kofler Block and are uncorrected. Elemental analyses were carried out in the Microanalytical Laboratory of the Faculty of Science, Cairo University. The IR 
spectra of compounds were recorded on a Bruker Tensor 37 Fourier Transform infrared 8400 spectrophotometer using potassium bromide pellets and frequencies are reported in $\mathrm{cm}^{-1}$. The ${ }^{1} \mathrm{H}$ - NMR spectra were recorded on a JEOL JNM ECA 500 MHZ instrument and chemical shifts $\delta_{\mathrm{H}}$ are given in ppm relative to tetramethylsilane used as internal standard. Mass spectra were recorded at $70 \mathrm{ev}$ with a GCMS-QP $1000 \mathrm{EX}$ spectrometer. Reactions were routinely followed by thin layer chromatography (TLC; Merck Kieselgel60-F254 precoated plastic plates). The spots were detected by iodine. 5-Aryl-7-hydrazino-2- phenylpyrazolo[1,5-c]pyrimidines 1 were prepared from the respective acetylenic $\beta$-diketones as described earlier [2,22].

\subsection{Synthesis of Compounds}

\subsubsection{5-Aryl-8-phenylpyrazolo[1,5-c]-1,2,4-triazolo[4,3- $a]$ pyrimidines $\mathbf{2 a - d}$}

A mixture of 5-aryl-7-hydrazino-2-phenylpyrazolo[1,5-c]pyrimidines (1a-d, $1 \mathrm{mmol})$ and formic acid $(10 \mathrm{~mL}, 99 \%)$ was heated under reflux for $10 \mathrm{~h}$. The mixture was evaporated under reduced pressure and the obtained residue was triturated with water, filtered, washed with EtOH and crystallized from EtOH to give the 5-aryl-8-phenylpyrazolo[1,5-c]-1,2,4-triazolo[4,3-a]pyrimidines 2a-d as colorless needles.

5,8-Diphenylpyrazolo[1,5-c]-1,2,4-triazolo[4,3-a]pyrimidine (2a). Yield 81\%, $0.25 \mathrm{~g}, \mathrm{mp} 245-246{ }^{\circ} \mathrm{C}$; IR $\left(v_{\max }, \mathrm{cm}^{-1}\right)$ : 1649 (pyrazole ring $\mathrm{C}=\mathrm{N}$ ), 1580 (triazole ring $\mathrm{C}=\mathrm{N}$ ), and $1476(\mathrm{C}=\mathrm{C})$; ${ }^{1} \mathrm{H}-\mathrm{NMR}$ $\left(\mathrm{CDCl}_{3}, \delta_{\mathrm{H}}, \mathrm{ppm}\right): 6.91(\mathrm{~s}, 1 \mathrm{H}$, pyrazole-H), $7.43(\mathrm{~s}, 1 \mathrm{H}$, pyrimidine- $\mathrm{H}), 7.45-7.64(\mathrm{~m}, 8 \mathrm{H}$, aromatic$\mathrm{H}), 8.05\left(\mathrm{~d}, 2 \mathrm{H}\right.$, aromatic-H) and $8.53\left(\mathrm{~s}, 1 \mathrm{H}\right.$, triazole-H); MS, $m / z(\%)=312\left(\mathrm{M}^{+}+1,100,\right), 285$ $\left(\mathrm{M}^{+}-\mathrm{CN}, 4\right), 283\left(\mathrm{M}^{+}-\mathrm{N}_{2}, 33\right), 257\left(\mathrm{M}^{+}-\mathrm{CN}_{3}, 3\right), 255\left(\mathrm{M}^{+}-\mathrm{CH}_{2} \mathrm{~N}_{3}, 16\right)$ and $227\left(\mathrm{M}^{+}-\mathrm{CH}_{2} \mathrm{~N}_{5}, 8\right)$; Anal. Calc. for $\mathrm{C}_{19} \mathrm{H}_{13} \mathrm{~N}_{5}$ (311.34): C, 73.30; H, 4.21; N, 22.49\%, found: C, 73.27; H, 4.22; N, 22.53\%.

8-Phenyl-5-p-tolylpyrazolo[1,5-c]-1,2,4-triazolo[4,3-a]pyrimidine (2b). Yield 76\%, $0.25 \mathrm{~g}, \mathrm{mp}$ 247-248 ${ }^{\circ} \mathrm{C}$; IR $\left(v_{\max }, \mathrm{cm}^{-1}\right)$ : 1643 (pyrazole ring $\mathrm{C}=\mathrm{N}$ ), 1577 (triazole ring $\mathrm{C}=\mathrm{N}$ ), and $1454(\mathrm{C}=\mathrm{C}$ ); ${ }^{1} \mathrm{H}$ NMR (DMSO-d6, $\left.\delta_{\mathrm{H}}, \mathrm{ppm}\right): 2.39(\mathrm{~s}, 3 \mathrm{H}, \mathrm{CH} 3), 7.03(\mathrm{~d}, 2 \mathrm{H}$, aromatic-H), $7.26(\mathrm{~s}, 1 \mathrm{H}$, pyrazole-H),7.39-7.51 (m, 5H, aromatic-H), $7.44(\mathrm{~s}, 1 \mathrm{H}$, pyrimidine-H), $7.65(\mathrm{~d}, 2 \mathrm{H}$, aromatic-H) and $8.96\left(\mathrm{~s}, 1 \mathrm{H}\right.$, triazole-H); MS, $m / z(\%)=327\left(\mathrm{M}^{+}+2,17\right), 325\left(\mathrm{M}^{+}, 100\right), 297\left(\mathrm{M}^{+}-\mathrm{N} 2,24\right), 282$ $\left(\mathrm{M}^{+}-\mathrm{CH}_{3} \mathrm{~N}_{2}, 8\right), 255\left(\mathrm{M} .+-\mathrm{C}_{2} \mathrm{H}_{4} \mathrm{~N}_{3}, 7\right)$ and $227\left(\mathrm{M}^{+}-\mathrm{C}_{2} \mathrm{H}_{4} \mathrm{~N}_{5}, 10\right)$; Anal. Calc. for $\mathrm{C}_{20} \mathrm{H}_{15} \mathrm{~N}_{5}(325.37)$ : C, $73.83 ; \mathrm{H}, 4.65 ; \mathrm{N}, 21.52 \%$, found: $\mathrm{C}, 73.87 ; \mathrm{H}, 4.62 ; \mathrm{N}, 21.55 \%$.

5-(p-Methoxyphenyl)-8-phenylpyrazolo[1,5-c]-1,2,4-triazolo[4,3-a]pyrimidine (2c). Yield 74\%, $0.25 \mathrm{~g}$, mp 237-238 ${ }^{\circ} \mathrm{C}$; IR $\left(v_{\max }, \mathrm{cm}^{-1}\right)$ : 1643 (pyrazole ring $\mathrm{C}=\mathrm{N}$ ), 1587 (triazole ring $\mathrm{C}=\mathrm{N}$ ), and 1454 $(\mathrm{C}=\mathrm{C}) ;{ }^{1} \mathrm{H}$ NMR $\left(\mathrm{CDCl} 3, \delta_{\mathrm{H}}, \mathrm{ppm}\right): 3.92\left(\mathrm{~s}, 3 \mathrm{H}, \mathrm{CH}_{3}\right), 6.93(\mathrm{~s}, 1 \mathrm{H}$, pyrazole-H), $7.10(\mathrm{~d}, 2 \mathrm{H}$, aromatic$\mathrm{H}), 7.40-7.48(\mathrm{~m}, 3 \mathrm{H}$, aromatic-H), $7.44(\mathrm{~s}, 1 \mathrm{H}$, pyrimidine- $\mathrm{H}), 7.57(\mathrm{~d}, 2 \mathrm{H}$, aromatic- $\mathrm{H}), 8.05(\mathrm{~d}$, $2 \mathrm{H}$, aromatic- $\mathrm{H})$ and $8.55\left(\mathrm{~s}, 1 \mathrm{H}\right.$, triazole-H); MS, $m / z(\%)=343\left(\mathrm{M}^{+}+2,15\right), 341\left(\mathrm{M}^{+}, 100\right), 326$ $\left(\mathrm{M}^{+}-\mathrm{CH}_{3}, 3\right), 313\left(\mathrm{M}^{+}-\mathrm{N}_{2}, 10\right), 299\left(\mathrm{M}^{+}-\mathrm{CH}_{2} \mathrm{~N}_{2}, 8\right), 285\left(\mathrm{M}^{+}-\mathrm{C}_{2} \mathrm{H}_{4} \mathrm{~N}_{2}, 2\right)$ and $270\left(\mathrm{M}^{+}-\mathrm{C}_{2} \mathrm{H}_{3} \mathrm{~N}_{2} \mathrm{O}\right.$, 11); Anal. Calc. for $\mathrm{C}_{20} \mathrm{H}_{15} \mathrm{~N}_{5} \mathrm{O}$ (341.37): C, 70.37; H, 4.43; N, 20.52\%, found: C, 70.40; H, 4.40; N, 20.55\%. 
5-(p-Chlorophenyl)-8-phenylpyrazolo[1,5-c]-1,2,4-triazolo[4,3-a]pyrimidine (2d). Yield 71\%, $0.25 \mathrm{~g}$, mp 299-300 ${ }^{\circ} \mathrm{C}$; IR $\left(v_{\max }, \mathrm{cm}^{-1}\right)$ : 1643 (pyrazole ring $\mathrm{C}=\mathrm{N}$ ), 1583 (triazole ring $\mathrm{C}=\mathrm{N}$ ), and 1467 $(\mathrm{C}=\mathrm{C}) ;{ }^{1} \mathrm{H}$ NMR (DMSO- $\left.d_{6}, \delta_{\mathrm{H}}, \mathrm{ppm}\right): 7.33$ (s, $1 \mathrm{H}$, pyrazole-H), 7.43 (s, $1 \mathrm{H}$, pyrimidine-H), 7.44-7.52 (m, 3H, aromatic- $\mathrm{H}), 7.66(\mathrm{~d}, 2 \mathrm{H}$, aromatic- $\mathrm{H}), 7.79(\mathrm{~d}, 2 \mathrm{H}$, aromatic- $\mathrm{H}), 8.04(\mathrm{~d}, 2 \mathrm{H}$, aromatic-H) and $9.00\left(\mathrm{~s}, 1 \mathrm{H}\right.$, triazole-H); MS, $m / z(\%)=347\left(\mathrm{M}^{+}+1,52\right), 345\left(\mathrm{M}^{+}{ }^{+}-1,100\right), 319$ $\left(\mathrm{M}^{+}-\mathrm{HCN}, 8\right), 317\left(\mathrm{M}^{+}-\mathrm{HN}_{2}, 17\right), 289\left(\mathrm{M}^{+}-\mathrm{C}_{2} \mathrm{H}_{5} \mathrm{~N}_{2}, 6\right), 282\left(\mathrm{M}^{+}-\mathrm{HClN}_{2}, 13\right), 255\left(\mathrm{M}^{+}{ }^{+} \mathrm{C}_{2} \mathrm{H}_{4} \mathrm{ClN}_{2}\right.$, 16) and $227\left(\mathrm{M}^{+}-\mathrm{C}_{3} \mathrm{H}_{6} \mathrm{ClN}_{3}, 8\right)$; Anal. Calc. for $\mathrm{C}_{19} \mathrm{H}_{12} \mathrm{ClN}_{5}$ (345.79): C, 66.00; H, 3.50; N, 20.25\%, found: C, 59.98; H, 3.50; N, 20.22\%.

\subsubsection{5-Aryl-6-bromo-8-phenylpyrazolo[1,5-c]-1,2,4-triazolo[4,3-a]pyrimidines 3a-d}

A solution of bromine $(0.06 \mathrm{~mL}, 1.2 \mathrm{mmol})$ in acetic acid $(10 \mathrm{~mL})$ was gradually added to a suspension of 5-aryl-8-phenylpyrazolo[1,5-c]-1,2,4-triazolo[4,3-a]pyrimidines 2a-d (1 mmol) in acetic acid $(10 \mathrm{~mL})$ with stirring for $3 \mathrm{~h}$ at room temperature. The precipitated 5-aryl-6-bromo-8phenylpyrazolo[1,5-c]-1,2,4-triazolo-[4,3-a]pyrimidines 3a-d were filtered, washed with water, dried and crystallized from EtOH as colorless needles.

6-Bromo-5,8-diphenylpyrazolo[1,5-c]-1,2,4-triazolo[4,3-a]pyrimidine (3a). Yield 75\%, $0.30 \mathrm{~g}, \mathrm{mp}$ 235-236 ${ }^{\circ} \mathrm{C}$; IR $\left(v_{\max }, \mathrm{cm}^{-1}\right)$ : 1640 (pyrazole ring $\mathrm{C}=\mathrm{N}$ ), 1580 (triazole ring $\mathrm{C}=\mathrm{N}$ ), and $1455(\mathrm{C}=\mathrm{C}$ ); ${ }^{1} \mathrm{H}$ NMR $\left(\mathrm{CDCl}_{3}, \delta_{\mathrm{H}}, \mathrm{ppm}\right): 6.92(\mathrm{~s}, 1 \mathrm{H}$, pyrazole-H), $7.43-7.69(\mathrm{~m}, 8 \mathrm{H}$, aromatic-H), $8.14(\mathrm{~d}$, $2 \mathrm{H}$, aromatic-H) and $8.58\left(\mathrm{~s}, 1 \mathrm{H}\right.$, triazole-H); MS, $m / z(\%)=390\left(\mathrm{M}^{+}, 100\right), 362\left(\mathrm{M}^{+}-\mathrm{N}_{2}, 13\right), 310$ $\left(\mathrm{M}^{+}-\mathrm{Br}, 2\right), 282\left(\mathrm{M}^{+}-\mathrm{BrN}_{2}, 13\right), 255\left(\mathrm{M}^{+}-\mathrm{CHBrN}_{3}, 12\right)$ and $227\left(\mathrm{M}^{+}-\mathrm{CHBrN}_{5}, 6\right)$; Anal. Calc. for $\mathrm{C}_{19} \mathrm{H}_{12} \mathrm{BrN}_{5}$ (390.24): C, 58.48; H, 3.10; N, 17.95\%, found: C, 58.52; H, 3.08; N, 17.90\%.

6-Bromo-8-phenyl-5-p-tolylpyrazolo[1,5-c]-1,2,4-triazolo[4,3-a]pyrimidine (3b). Yield 75\%, $0.30 \mathrm{~g}$, mp 215-216 ${ }^{\circ} \mathrm{C}$; IR $\left(v_{\max }, \mathrm{cm}^{-1}\right)$ : 1636 (pyrazole ring $\left.\mathrm{C}=\mathrm{N}\right), 1578$ (triazole ring $\mathrm{C}=\mathrm{N}$ ), and 1421 $(\mathrm{C}=\mathrm{C}) ;{ }^{1} \mathrm{H}$ NMR $\left(\mathrm{CDCl}_{3}, \delta_{\mathrm{H}}, \mathrm{ppm}\right): 2.49\left(\mathrm{~s}, 3 \mathrm{H}, \underline{\mathrm{CH}}_{3}\right), 6.90(\mathrm{~s}, 1 \mathrm{H}$, pyrazole-H), 7.41-7.57 (m, 7H, aromatic-H), $8.14\left(\mathrm{~d}, 2 \mathrm{H}\right.$, aromatic-H) and $8.60\left(\mathrm{~s}, 1 \mathrm{H}\right.$, triazole-H); MS, $m / z(\%)=407\left(\mathrm{M}^{+}+3,8\right)$, $405\left(\mathrm{M}^{+}+1,100\right), 377\left(\mathrm{M}^{+}-\mathrm{HCN}, 8\right), 324\left(\mathrm{M}^{+}-\mathrm{Br}, 2\right), 281 \quad\left(\mathrm{M}^{+}-\mathrm{CH}_{3} \mathrm{BrN}_{2}, 6\right)$ and 254 $\left(\mathrm{M}^{+}-\mathrm{C}_{2} \mathrm{H}_{4} \mathrm{BrN}_{3}\right.$, 5); Anal. Calc. for $\mathrm{C}_{20} \mathrm{H}_{14} \mathrm{BrN}_{5}$ (404.26): C, 59.42; H, 3.49; N, 17.32\%, found: C, 59.39; H, 3.45; N, 17.27\%.

6-Bromo-5-(p-methoxyphenyl)-8-phenylpyrazolo[1,5-c]-1,2,4-triazolo[4,3-a]pyrimidine (3c). Yield $71 \%, 0.30 \mathrm{~g}, \mathrm{mp} 213-214{ }^{\circ} \mathrm{C}$; IR $\left(v_{\max }, \mathrm{cm}^{-1}\right)$ : 1632 (pyrazole ring $\left.\mathrm{C}=\mathrm{N}\right), 1587$ (triazole ring $\left.\mathrm{C}=\mathrm{N}\right)$, and $1427(\mathrm{C}=\mathrm{C}) ;{ }^{1} \mathrm{H}$ NMR $\left(\mathrm{CDCl}_{3}, \delta_{\mathrm{H}}, \mathrm{ppm}\right): 3.90\left(\mathrm{~s}, 3 \mathrm{H}, \mathrm{OCH}_{3}\right), 6.81(\mathrm{~s}, 1 \mathrm{H}$, pyrazole-H), $7.09(\mathrm{~d}$, $2 \mathrm{H}$, aromatic-H), 7.44-7.56 (m, 3H, aromatic-H), $7.59(\mathrm{~d}, 2 \mathrm{H}$, aromatic- $\mathrm{H}), 8.10(\mathrm{~d}, 2 \mathrm{H}$, aromatic$\mathrm{H})$ and $8.57\left(\mathrm{~s}, 1 \mathrm{H}\right.$, triazole-H); MS, $m / z(\%)=422\left(\mathrm{M}^{+}+2,100\right), 420\left(\mathrm{M}^{+}, 80\right), 405\left(\mathrm{M}^{+}-\mathrm{CH}_{3}, 3\right)$, $391\left(\mathrm{M}^{+}-\mathrm{HN}_{2}, 8\right), 378\left(\mathrm{M}^{+}-\mathrm{CH}_{2} \mathrm{~N}_{2}, 5\right), 350\left(\mathrm{M}^{+}-\mathrm{C}_{2} \mathrm{H}_{4} \mathrm{~N}_{3}, 6\right), 340\left(\mathrm{M}^{+}-\mathrm{Br}, 3\right), 313\left(\mathrm{M}^{+}{ }^{-} \mathrm{CHBrN}, 4\right)$, $281\left(\mathrm{M}^{+}-\mathrm{CH}_{3} \mathrm{BrN}_{2} \mathrm{O}, 5\right)$ and $269\left(\mathrm{M}^{+}{ }_{-}-\mathrm{C}_{2} \mathrm{H}_{3} \mathrm{BrN}_{2} \mathrm{O}, 13\right)$; Anal. Calc. for $\mathrm{C}_{20} \mathrm{H}_{14} \mathrm{BrN}_{5} \mathrm{O}$ (420.26): $\mathrm{C}$, 57.16; H, 3.36; N, 16.66\%, found: C, 57.20; H, 3.38; N, 16.70\%.

6-Bromo-5-(p-chlorophenyl)-8-phenylpyrazolo[1,5-c]-1,2,4-triazolo[4,3-a]pyrimidine (3d). Yield $71 \%, 0.30 \mathrm{~g}, \mathrm{mp} 238-239^{\circ} \mathrm{C}$; IR $\left(v_{\max }, \mathrm{cm}^{-1}\right)$ : 1637 (pyrazole ring $\left.\mathrm{C}=\mathrm{N}\right), 1580$ (triazole ring $\left.\mathrm{C}=\mathrm{N}\right)$, 
and $1414(\mathrm{C}=\mathrm{C}) ;{ }^{1} \mathrm{H}$ NMR $\left(\mathrm{CDCl}_{3}, \delta_{\mathrm{H}}, \mathrm{ppm}\right): 6.92(\mathrm{~s}, 1 \mathrm{H}$, pyrazole- $\mathrm{H}), 7.47-7.66(\mathrm{~m}, 7 \mathrm{H}$, aromatic-H), $8.14\left(\mathrm{~d}, 2 \mathrm{H}\right.$, aromatic-H) and $8.55\left(\mathrm{~s}, 1 \mathrm{H}\right.$, triazole-H); MS, $m / z(\%)=429\left(\mathrm{M}^{+}+4,3\right)$, $428\left(\mathrm{M}^{+}+3,38\right), 426\left(\mathrm{M}^{+}+1,100\right), 424\left(\mathrm{M}^{+}-1,76\right), 398\left(\mathrm{M}^{+}-\mathrm{HCN}, 11\right), 317\left(\mathrm{M}^{+}-\mathrm{BrN}_{2}, 8\right), 289$ $\left(\mathrm{M}^{+}-\mathrm{CH}_{2} \mathrm{BrN}_{3}, 8\right), 281\left(\mathrm{M}^{+}-\mathrm{HBrClN}_{2}, 15\right), 253\left(\mathrm{M}^{+}-\mathrm{C}_{2} \mathrm{H}_{5} \mathrm{BrClN}_{2}, 10\right)$ and $226\left(\mathrm{M}^{+}-\mathrm{C}_{3} \mathrm{H}_{6} \mathrm{BrClN}_{3}\right.$, 7); Anal. Calc. for $\mathrm{C}_{19} \mathrm{H}_{11} \mathrm{BrClN}_{5}$ (424.68): C, 53.74; H, 2.61; N, 16.49\%; found: C, 53.72; H, 2.57; N, $16.50 \%$.

\subsubsection{5-Aryl-6-iodo-8-phenylpyrazolo[1,5-c]-1,2,4-triazolo[4,3-a]pyrimidines 4a-d}

A solution of iodine monochloride $(0.2 \mathrm{~g}, 1.2 \mathrm{mmol})$ in acetic acid $(10 \mathrm{~mL})$ was gradually added to a suspension of 5- aryl-8-phenylpyrazolo[1,5-c]-1,2,4-triazolo[3,4-a]pyrimidines 2a-d (1mmol) in acetic acid $(10 \mathrm{~mL})$ with stirring for $3 \mathrm{~h}$ at room temperature. The reaction mixture was then poured onto crushed ice and the precipitated 5-aryl-6-iodo-8-phenylpyrazolo[1,5,c]-1,2,4-triazolo[3,4a]pyrimidines 4a-d were filtered, washed with water, dried and crystallized from EtOH as colorless needles.

6-Iodo-5,8-diphenylpyrazolo[1,5-c]-1,2,4-triazolo[4,3-a]pyrimidine (4a). Yield 91\%, $0.40 \mathrm{~g}, \mathrm{mp}$ 281-282 ${ }^{\circ} \mathrm{C}$; IR ( $\left.v_{\max }, \mathrm{cm}^{-1}\right): 1640$ (pyrazole ring $\mathrm{C}=\mathrm{N}$ ), 1575 (triazole ring $\mathrm{C}=\mathrm{N}$ ), and 1405 $(\mathrm{C}=\mathrm{C}) ;{ }^{1} \mathrm{H}$ NMR $\left(\mathrm{CDCl}_{3}, \delta_{\mathrm{H}}, \mathrm{ppm}\right): 6.94$ (s, 1H, pyrazole-H), 7.50-7.69 (m, 8H, aromatic-H), $8.10\left(\mathrm{~d}, 2 \mathrm{H}\right.$, aromatic-H) and $8.61\left(\mathrm{~s}, 1 \mathrm{H}\right.$, triazole-H); MS, $m / z(\%)=437\left(\mathrm{M}^{+}, 100\right), 409\left(\mathrm{M}^{+}-\mathrm{N}_{2}\right.$, 5), $310\left(\mathrm{M}^{+}-\mathrm{I}, 7\right), 282\left(\mathrm{M}^{+}-\mathrm{IN}_{2}, 10\right), 242\left(\mathrm{M}^{+}-\mathrm{C}_{2} \mathrm{H}_{2} \mathrm{IN}_{3}, 4\right)$ and $227\left(\mathrm{M}^{+}-\mathrm{C}_{3} \mathrm{H}_{5} \mathrm{IN}_{3}, 8\right)$; Anal. Calc. for $\mathrm{C}_{19} \mathrm{H}_{12} \mathrm{IN}_{5}$ (437.24): C, 52.19; H, 2.77; N, 16.02\%, found: C, 52.17; H, 2.80; N, $16.00 \%$.

6-Iodo-8-phenyl-5-p-tolylpyrazolo[1,5-c]-1,2,4-triazolo[4,3-a]pyrimidine (4b). Yield 89\%, $0.40 \mathrm{~g}$, mp 245-246 ${ }^{\circ} \mathrm{C}$; IR $\left(v_{\max }, \mathrm{cm}^{-1}\right)$ : 1633 (pyrazole ring $\mathrm{C}=\mathrm{N}$ ), 1576 (triazole ring $\mathrm{C}=\mathrm{N}$ ), and 1416 $(\mathrm{C}=\mathrm{C}) ;{ }^{1} \mathrm{H} \mathrm{NMR}\left(\mathrm{CDCl}_{3}, \delta_{\mathrm{H}}, \mathrm{ppm}\right): 2.49\left(\mathrm{~s}, 3 \mathrm{H}, \underline{\mathrm{CH}}_{3}\right), 6.90(\mathrm{~s}, 1 \mathrm{H}$, pyrazole-H), 7.41-7.69 (m, 7H, aromatic-H), $8.08(\mathrm{~d}, 2 \mathrm{H}$, aromatic- $\mathrm{H})$ and $8.60(\mathrm{~s}, 1 \mathrm{H}$, triazole- $\mathrm{H}) ; \mathrm{MS}, m / z(\%)=453\left(\mathrm{M}^{+}+2,9\right)$, $452\left(\mathrm{M}^{+}+1,100\right), 423\left(\mathrm{M}^{+}-\mathrm{N}_{2}, 5\right), 325\left(\mathrm{M}^{+}+1-\mathrm{I}, 12\right), 296\left(\mathrm{M}^{+}-\mathrm{IN}_{2}, 9\right)$ and $269\left(\mathrm{M}^{+}-\mathrm{CHIN}_{3}, 10\right)$; Anal. Calc. for $\mathrm{C}_{20} \mathrm{H}_{14} \mathrm{IN}_{5}$ (451.26): C, 53.23; H, 3.13; N, 15.52\%, found: C, 53.27; H, 3.15; N, $15.50 \%$.

6-Iodo-5-(p-methoxyphenyl)-8-phenylpyrazolo[1,5-c]-1,2,4-triazolo[4,3-a]pyrimidine (4c). Yield 85\%, $0.40 \mathrm{~g}$, mp $225-226{ }^{\circ} \mathrm{C}$; IR $\left(v_{\max }, \mathrm{cm}^{-1}\right)$ : 1623 (pyrazole ring $\mathrm{C}=\mathrm{N}$ ), 1572 (triazole ring $\mathrm{C}=\mathrm{N}$ ), and $1458(\mathrm{C}=\mathrm{C}) ;{ }^{1} \mathrm{H}$ NMR $\left(\mathrm{CDCl}_{3}, \delta_{\mathrm{H}}, \mathrm{ppm}\right): 3.93\left(\mathrm{~s}, 3 \mathrm{H}, \mathrm{OCH}_{3}\right), 6.86(\mathrm{~s}, 1 \mathrm{H}$, pyrazole- $\mathrm{H}), 7.12(\mathrm{~d}, 2 \mathrm{H}$, aromatic-H), 7.48-7.51 (m, 3H, aromatic-H), $7.61(\mathrm{~d}, 2 \mathrm{H}$, aromatic-H), $8.10(\mathrm{~d}, 2 \mathrm{H}$, aromatic-H) and $8.61\left(\mathrm{~s}, 1 \mathrm{H}\right.$, triazole-H); MS, $m / z(\%)=470\left(\mathrm{M}^{+}+3,3\right), 468\left(\mathrm{M}^{+}+1,100\right), 439\left(\mathrm{M}^{+}-\mathrm{N}_{2}, 6\right), 341$ $\left(\mathrm{M}^{++}+1-\mathrm{I}, 12\right), 313\left(\mathrm{M}^{+}-\mathrm{CHIN}, 4\right), 285\left(\mathrm{M}^{+}-\mathrm{CHIN}_{3}, \quad 7\right), 269\left(\mathrm{M}^{+}-\mathrm{C}_{2} \mathrm{H}_{3} \mathrm{IN}_{2} \mathrm{O}, 12\right)$ and 255 $\left(\mathrm{M}^{+}-\mathrm{C}_{2} \mathrm{H}_{3} \mathrm{IN}_{3} \mathrm{O}\right.$, 4); Anal. Calc. for $\mathrm{C}_{20} \mathrm{H}_{14} \mathrm{IN}_{5} \mathrm{O}$ (467.26): C, 51.41; H, 3.02; N, 14.99\%, found: C, 51.44; H, 3.00; N, 15.02\%.

5-(p-Chlorophenyl)-6-iodo-8-phenylpyrazolo[1,5-c]-1,2,4-triazolo[4,3-a]pyrimidine (4d). Yield 85\%, $0.40 \mathrm{~g}, \mathrm{mp} 270-27{ }^{\circ} \mathrm{C}$; IR $\left(\mathrm{v}_{\max }, \mathrm{cm}^{-1}\right): 1632$ (pyrazole ring $\left.\mathrm{C}=\mathrm{N}\right), 1576$ (triazole ring $\mathrm{C}=\mathrm{N}$ ), and $1410(\mathrm{C}=\mathrm{C}) ;{ }^{1} \mathrm{H}$ NMR (DMSO- $\left.d_{6}, \delta_{\mathrm{H}}, \mathrm{ppm}\right): 7.02(\mathrm{~s}, 1 \mathrm{H}$, pyrazole-H), 7.49-7.54 (m, 3H, 
aromatic-H), $7.65(\mathrm{~d}, 2 \mathrm{H}$, aromatic- $\mathrm{H}), 7.80(\mathrm{~d}, 2 \mathrm{H}$, aromatic- $\mathrm{H}), 7.92(\mathrm{~d}, 2 \mathrm{H}$, aromatic- $\mathrm{H})$ and 9.04 (s, 1H, triazole-H); MS, $m / z(\%)=475\left(\mathrm{M}^{+}+3,3\right), 472\left(\mathrm{M}^{+}, 100\right), 443\left(\mathrm{M}^{+}-\mathrm{HN}_{2}, 3\right), 345\left(\mathrm{M}^{+}-\mathrm{I}, 7\right)$, $309\left(\mathrm{M}^{+}\right.$-ClI, 2), $289\left(\mathrm{M}^{+}-\mathrm{CH}_{2} \mathrm{IN}_{3}, 5\right), 282\left(\mathrm{M}^{+}-\mathrm{CHClIN}, 8\right)$ and $241\left(\mathrm{M}^{+}-\mathrm{C}_{2} \mathrm{H}_{2} \mathrm{ClIN}_{3}\right.$, 9); Anal. Calc. for $\mathrm{C}_{19} \mathrm{H}_{11} \mathrm{ClIN}_{5}$ (471.68): C, 48.38; H, 2.35; N, 14.85\%, found: C, 48.40; H, 2.40; N, 14.90\%.

\subsubsection{5-Aryl-6-nitro-8-phenylpyrazolo[1,5-c]-1,2,4-triazolo[4,3-a]pyrimidines 5a-d}

A mixture of nitric acid $(\mathrm{d} 1.41,1 \mathrm{~mL})$ and sulfuric acid $(\mathrm{d} 1.84,1 \mathrm{~mL})$ in glacial acetic acid $(10 \mathrm{~mL})$ was gradually added to a suspension of 5-aryl-8-phenylpyrazolo[1,5-c]-1,2,4-triazolo[4,3a]pyrimidines $\mathbf{2 a - d}(1 \mathrm{mmol})$ in glacial acetic acid $(10 \mathrm{~mL})$ with stirring for $3 \mathrm{~h}$ at room temperature. The reaction mixture was then poured onto cold water with stirring and the yellow precipitated solids were filtered, washed with cold water, dried and crystallized from EtOH to give the title compounds 5a-d as yellow needles.

6-Nitro-5,8-diphenylpyrazolo[1,5-c]-1,2,4-triazolo[4,3-a]pyrimidine (5a). Yield 83\%, $0.30 \mathrm{~g}, \mathrm{mp}$ 241-242 ${ }^{\circ} \mathrm{C}$; IR $\left(v_{\max }, \mathrm{cm}^{-1}\right)$ : 1649 (pyrazole ring $\left.\mathrm{C}=\mathrm{N}\right), 1579$ (triazole ring $\left.\mathrm{C}=\mathrm{N}\right)$, and $1462(\mathrm{C}=\mathrm{C})$; ${ }^{1} \mathrm{H}$ NMR $\left(\mathrm{CDCl}_{3}, \delta_{\mathrm{H}}, \mathrm{ppm}\right): 6.93(\mathrm{~s}, 1 \mathrm{H}$, pyrazole-H), 7.40-7.44 (m, 6H, aromatic-H), $7.62(\mathrm{~d}$, $2 \mathrm{H}$, aromatic- $\mathrm{H}), 8.03\left(\mathrm{~d}, 2 \mathrm{H}\right.$, aromatic-H) and $8.52(\mathrm{~s}, 1 \mathrm{H}$, triazole- $\mathrm{H}) ; \mathrm{MS}, m / z(\%)=356\left(\mathrm{M}^{+}, 7\right)$, $326\left(\mathrm{M}^{+}-\mathrm{H}_{2} \mathrm{~N}_{2}, 4\right), 311\left(\mathrm{M}^{+}+1-\mathrm{NO}_{2}, 100\right), 283\left(\mathrm{M}^{+}-\mathrm{CHN}_{2} \mathrm{O}_{2}, 27\right), 271\left(\mathrm{M}^{+}+1-\mathrm{CN}_{3} \mathrm{O}_{2}, 2\right)$ and 255 $\left(\mathrm{M}^{+}-\mathrm{CHN}_{4} \mathrm{O}_{2}\right.$, 13); Anal. Calc. for $\mathrm{C}_{19} \mathrm{H}_{12} \mathrm{~N}_{6} \mathrm{O}_{2}$ (356.34): C, 64.04; H, 3.39; N, 23.58\%, found: C, $64.00 ; \mathrm{H}, 3.40 ; \mathrm{N}, 23.60 \%$.

6-Nitro-8-phenyl-5-p-tolylpyrazolo[1,5-c]-1,2,4-triazolo[4,3-a]pyrimidine (5b). Yield 81\%, $0.30 \mathrm{~g}, \mathrm{mp}$ 243-244 ${ }^{\circ} \mathrm{C}$; IR $\left(v_{\max }, \mathrm{cm}^{-1}\right)$ : 1643 (pyrazole ring $\mathrm{C}=\mathrm{N}$ ), 1578 (triazole ring $\mathrm{C}=\mathrm{N}$ ), and $1415(\mathrm{C}=\mathrm{C}$ ); ${ }^{1} \mathrm{H}$ NMR $\left(\mathrm{CDCl}_{3}, \delta_{\mathrm{H}}, \mathrm{ppm}\right): 2.47\left(\mathrm{~s}, 3 \mathrm{H}, \underline{\mathrm{CH}_{3}}\right), 6.90(\mathrm{~s}, 1 \mathrm{H}$, pyrazole-H), 7.37-7.45 (m, 5H, aromatic$\mathrm{H}), 7.51(\mathrm{~d}, 2 \mathrm{H}$, aromatic-H), $8.03(\mathrm{~d}, 2 \mathrm{H}$, aromatic-H) and $8.54(\mathrm{~s}, 1 \mathrm{H}$, triazole-H); $\mathrm{MS}, m / z(\%)=$ $370\left(\mathrm{M}^{+}, 4\right), 340\left(\mathrm{M}^{+}-\mathrm{H}_{2} \mathrm{~N}_{2}, 2\right), 325\left(\mathrm{M}^{++}+1-\mathrm{NO}_{2}, 100\right), 309\left(\mathrm{M}^{+}-\mathrm{CH}_{3} \mathrm{NO}_{2}, 1\right), 297\left(\mathrm{M}^{+}-\mathrm{CHN}_{2} \mathrm{O}_{2}, 22\right)$ and $269\left(\mathrm{M}^{+}-\mathrm{C}_{2} \mathrm{H}_{3} \mathrm{~N}_{3} \mathrm{O}_{2}\right.$, 9); Anal. Calc. for $\mathrm{C}_{20} \mathrm{H}_{14} \mathrm{~N}_{6} \mathrm{O}_{2}$ (370.36): C, 64.86; $\mathrm{H}, 3.81$; $\mathrm{N}, 22.69 \%$, found: C, 64.90; H, 3.80; N, 22.72\%.

5-(p-Methoxyphenyl)-6-nitro-8-phenylpyrazolo[1,5-c]-1,2,4-triazolo[4,3-a]pyrimidine (5c), Yield $77 \%, 0.30 \mathrm{~g}, \mathrm{mp} 250-251{ }^{\circ} \mathrm{C}$; IR $\left(\mathrm{v}_{\max }, \mathrm{cm}^{-1}\right)$ : 1637 (pyrazole ring $\left.\mathrm{C}=\mathrm{N}\right), 1585$ (triazole ring $\left.\mathrm{C}=\mathrm{N}\right)$, and $1420(\mathrm{C}=\mathrm{C}) ;{ }^{1} \mathrm{H}$ NMR $\left(\mathrm{CDCl}_{3}, \delta_{\mathrm{H}}, \mathrm{ppm}\right): 3.89\left(\mathrm{~s}, 3 \mathrm{H}, \underline{\mathrm{OCH}}_{3}\right), 6.97(\mathrm{~s}, 1 \mathrm{H}$, pyrazole-H), 7.45-7.47 $(\mathrm{m}, 3 \mathrm{H}$, aromatic- $\mathrm{H}), 7.62(\mathrm{~d}, 2 \mathrm{H}$, aromatic- $\mathrm{H}), 7.79(\mathrm{~d}, 2 \mathrm{H}$, aromatic- $\mathrm{H}), 8.02(\mathrm{~d}, 2 \mathrm{H}$, aromatic-H) and $8.75\left(\mathrm{~s}, 1 \mathrm{H}\right.$, triazole-H); $\mathrm{MS}, m / z(\%)=388\left(\mathrm{M}^{+}+2,15\right), 387\left(\mathrm{M}^{+}+1,100\right), 356\left(\mathrm{M}^{+}-\mathrm{CH}_{2} \mathrm{O}, 7\right)$, $339\left(\mathrm{M}^{+}-\mathrm{HNO}_{2}, 7\right), 312\left(\mathrm{M}^{+}-\mathrm{CH}_{2} \mathrm{~N}_{2} \mathrm{O}_{2}, 4\right), 310\left(\mathrm{M}^{+}-\mathrm{CH}_{4} \mathrm{~N}_{2} \mathrm{O}_{2}, 11\right), 284\left(\mathrm{M}^{+}{ }^{+}-\mathrm{C}_{3} \mathrm{H}_{6} \mathrm{~N}_{2} \mathrm{O}_{2}, 5\right)$ and 251 $\left(\mathrm{M}^{+}-\mathrm{C}_{7} \mathrm{H}_{7} \mathrm{~N}_{2} \mathrm{O}\right.$, 16); Anal. Calc. for $\mathrm{C}_{20} \mathrm{H}_{14} \mathrm{~N}_{6} \mathrm{O}_{3}$ (386.36): C, 62.17; H, 3.65; N, 21.75\%, found: C, $62.20 ; \mathrm{H}, 3.61 ; \mathrm{N}, 21.73 \%$.

5-(p-Chlorophenyl)-6-nitro-8-phenylpyrazolo[1,5-c]-1,2,4-triazolo[4,3-a]pyrimidine $\quad(5 \mathbf{d}) . \quad$ Yield $77 \%, 0.30 \mathrm{~g}, \mathrm{mp} 306-307{ }^{\circ} \mathrm{C}$; IR $\left(v_{\max }, \mathrm{cm}^{-1}\right)$ : 1641 (pyrazole ring $\left.\mathrm{C}=\mathrm{N}\right), 1583$ (triazole ring $\left.\mathrm{C}=\mathrm{N}\right)$, and $1416(\mathrm{C}=\mathrm{C}) ;{ }^{1} \mathrm{H}$ NMR (DMSO- $\left.d_{6}, \delta_{\mathrm{H}}, \mathrm{ppm}\right): 7.33(\mathrm{~s}, 1 \mathrm{H}$, pyrazole- $\mathrm{H}), 7.43(\mathrm{t}, 1 \mathrm{H}$, aromatic- $\mathrm{H})$, $7.50(\mathrm{t}, 2 \mathrm{H}$, aromatic-H), $7.66(\mathrm{~d}, 2 \mathrm{H}$, aromatic-H), $7.79(\mathrm{~d}, 2 \mathrm{H}$, aromatic- $\mathrm{H}), 8.03(\mathrm{~d}, 2 \mathrm{H}$, aromatic-H) 
and $8.99\left(\mathrm{~s}, 1 \mathrm{H}\right.$, triazole-H); MS, $m / z(\%)=391\left(\mathrm{M}^{+}, 3\right), 349\left(\mathrm{M}^{+}-\mathrm{CH}_{2} \mathrm{~N}_{2}, 3\right), 347\left(\mathrm{M}^{+}{ }^{-} \mathrm{N}_{2} \mathrm{O}, 46\right)$, $345\left(\mathrm{M}^{+}{ }^{-} \mathrm{NO}_{2}, 100\right), 317\left(\mathrm{M}^{+}{ }^{-} \mathrm{N}_{3} \mathrm{O}_{2}, 17\right), 289\left(\mathrm{M}^{+}-\mathrm{C}_{2} \mathrm{H}_{4} \mathrm{~N}_{3} \mathrm{O}_{2}, 7\right), 282\left(\mathrm{M}^{+}{ }^{-} \mathrm{C}_{6} \mathrm{H}_{5} \mathrm{O}_{2}, 15\right), 254\left(\mathrm{M}^{+}{ }_{-}\right.$ $\mathrm{C}_{7} \mathrm{H}_{4} \mathrm{ClN}$, 22) and $227\left(\mathrm{M}^{+}-\mathrm{C}_{7} \mathrm{H}_{3} \mathrm{ClN}_{3}, 12\right)$; Anal. Calc. for $\mathrm{C}_{19} \mathrm{H}_{11} \mathrm{ClN}_{6} \mathrm{O}_{2}$ (390.78): C, 58.40; H, 2.84; N, 21.51\%, found: C, 58.44; H, 2.80; N, 21.53\%.

\subsubsection{5-Aryl-7-formylhydrazino-2-phenylpyrazolo[1,5-c]pyrimidines 6a-d}

A suspension of 5-aryl-7-hydrazino-2-phenylpyrazolo[1,5-c]pyrimidines (1a-d, 1mmol) and ethyl formate $(5 \mathrm{~mL})$ was heated under reflux for $3 \mathrm{~h}$. The product which separated upon cooling was filtered, washed with EtOH and crystallized from EtOH to give the title compounds 6a-d as colorless needles.

7-Formylhydrazino-2,5-Diphenylpyrazolo[1,5-c]pyrimidine (6a). Yield 76\%, $0.25 \mathrm{~g}, \mathrm{mp} 177-178{ }^{\circ} \mathrm{C}$; IR (vmax, $\left.\mathrm{cm}^{-1}\right)$ : $3368(\mathrm{NH}), 1700(\mathrm{C}=\mathrm{O}), 1624$ (pyrazole ring $\left.\mathrm{C}=\mathrm{N}\right), 1568$ (pyrimidine ring $\mathrm{C}=\mathrm{N}$ ), and $1455(\mathrm{C}=\mathrm{C}) ;{ }^{1} \mathrm{H}$ NMR $\left(\mathrm{CDCl}_{3}, \delta_{\mathrm{H}}, \mathrm{ppm}\right): 4.80(\mathrm{~s}, 2 \mathrm{H}$, exchangeable $2 \mathrm{NH}), 6.72(\mathrm{~s}, 1 \mathrm{H}$, pyrazole-H), $7.29(\mathrm{~s}, 1 \mathrm{H}$, pyrimidine-H), 7.30-7.49 $(\mathrm{m}, 8 \mathrm{H}$, aromatic- $\mathrm{H}), 7.98(\mathrm{~d}, 2 \mathrm{H}$, aromatic- $\mathrm{H})$ and $8.08(\mathrm{~s}, 1 \mathrm{H}$, $\underline{\mathrm{CHO}}) ; \mathrm{MS}, m / z(\%)=330\left(\mathrm{M}^{+}+1,2\right), 302\left(\mathrm{M}^{+}+1-\mathrm{CO}, 100\right), 286\left(\mathrm{M}^{+}-\mathrm{CHNO}, 23\right), 272\left(\mathrm{M}^{+}{ }^{+}-\mathrm{CHN}_{2} \mathrm{O}\right.$, 81), $257\left(\mathrm{M}^{+}-\mathrm{C}_{2} \mathrm{H}_{4} \mathrm{~N}_{2} \mathrm{O}, 2\right), 244\left(\mathrm{M}^{+}-\mathrm{C}_{2} \mathrm{H}_{3} \mathrm{~N}_{3} \mathrm{O}, 17\right)$ and $228\left(\mathrm{M}^{+}-\mathrm{C}_{2} \mathrm{H}_{5} \mathrm{~N}_{4} \mathrm{O}, 6\right)$; Anal. Calc. for $\mathrm{C}_{19} \mathrm{H}_{15} \mathrm{~N}_{5} \mathrm{O}$ (329.36): C, 69.29; H, 4.59; N, 21.26\%, found: C, 69.30; H, 4.62; N, 21.30\%.

7-Formylhydrazino-2-phenyl-5-p-tolylpyrazolo[1,5-c]pyrimidine (6b). Yield 71\%, $0.25 \mathrm{~g}, \mathrm{mp}$ 132-133 ${ }^{\circ} \mathrm{C}$; IR $\left(v_{\max }, \mathrm{cm}^{-1}\right)$ : $3315(\mathrm{NH}), 1701(\mathrm{C}=\mathrm{O}), 1610$ (pyrazole ring $\mathrm{C}=\mathrm{N}$ ), 1572 (pyrimidine ring $\mathrm{C}=\mathrm{N})$, and $1447(\mathrm{C}=\mathrm{C}) ;{ }^{1} \mathrm{H} \mathrm{NMR}\left(\mathrm{CDCl}_{3}, \delta_{\mathrm{H}}, \mathrm{ppm}\right): 2.42\left(\mathrm{~s}, 3 \mathrm{H}, \underline{\mathrm{CH}}_{3}\right), 4.77(\mathrm{~s}, 2 \mathrm{H}$, exchangeable $2 \mathrm{NH}), 6.70(\mathrm{~s}, 1 \mathrm{H}$, pyrazole-H), $7.28(\mathrm{~s}, 1 \mathrm{H}$, pyrimidine-H), 7.29-7.49 (m, 7H, aromatic-H), $7.96(\mathrm{~d}$, $2 \mathrm{H}$, aromatic- $\mathrm{H})$ and $7.98(\mathrm{~s}, 1 \mathrm{H}, \underline{\mathrm{CHO}}) ; \mathrm{MS}, m / z(\%)=344\left(\mathrm{M}^{+}+1,2\right), 316\left(\mathrm{M}^{+}+1-\mathrm{CO}, 100\right)$, $300\left(\mathrm{M}^{+}-\mathrm{CHNO}, 33\right), 286\left(\mathrm{M}^{+}-\mathrm{CHN}_{2} \mathrm{O}, 85\right), 270\left(\mathrm{M}^{+}-\mathrm{C}_{2} \mathrm{H}_{5} \mathrm{~N}_{2} \mathrm{O}, 4\right), 259\left(\mathrm{M}^{+}-\mathrm{C}_{2} \mathrm{H}_{2} \mathrm{~N}_{3} \mathrm{O}, 7\right)$ and 227 $\left(\mathrm{M}^{+}-\mathrm{C}_{8} \mathrm{H}_{6} \mathrm{~N}, 10\right)$; Anal. Calc. for $\mathrm{C}_{20} \mathrm{H}_{17} \mathrm{~N}_{5} \mathrm{O}$ (343.38): C, 69.96; H, 4.99; N, 20.40\%, found: C, $70.02 ; \mathrm{H}, 5.02 ; \mathrm{N}, 20.44 \%$

7-Formylhydrazino-5-(p-methoxyphenyl)-2-phenylpyrazolo[1,5-c]pyrimidine (6c). Yield 69\%, $0.25 \mathrm{~g}$, mp 157-158 ${ }^{\circ} \mathrm{C}$; IR $\left(v_{\max }, \mathrm{cm}^{-1}\right): 3265(\mathrm{NH}), 1708(\mathrm{C}=\mathrm{O}), 1667$ (pyrazole ring $\left.\mathrm{C}=\mathrm{N}\right), 1585$ (pyrimidine ring $\mathrm{C}=\mathrm{N})$, and $1448(\mathrm{C}=\mathrm{C}) ;{ }^{1} \mathrm{H} \mathrm{NMR}\left(\mathrm{CDCl}_{3}, \delta_{\mathrm{H}}, \mathrm{ppm}\right): 3.87\left(\mathrm{~s}, 3 \mathrm{H}, \underline{\mathrm{OCH}}_{3}\right), 4.73$ (s, $2 \mathrm{H}$, exchangeable $2 \mathrm{NH}), 6.68(\mathrm{~s}, 1 \mathrm{H}$, pyrazole-H), $7.19(\mathrm{~s}, 1 \mathrm{H}$, pyrimidine- $\mathrm{H}), 7.20-7.46(\mathrm{~m}, 3 \mathrm{H}$, aromatic- $\mathrm{H}), 7.95-7.97(\mathrm{~m}, 4 \mathrm{H}$, aromatic-H), $7.98(\mathrm{~d}, 2 \mathrm{H}$, aromatic-H) and $8.00(\mathrm{~s}, 1 \mathrm{H}, \underline{\mathrm{CHO}})$; MS, $m / z(\%)=361\left(\mathrm{M}^{+}+2,5\right), 359\left(\mathrm{M}^{+}, 30\right), 332\left(\mathrm{M}^{+}+1-\mathrm{CO}, 100\right), 316\left(\mathrm{M}^{+}-\mathrm{CHNO}, 23\right), 302$ $\left(\mathrm{M}^{+}-\mathrm{CHN}_{2} \mathrm{O}, 78\right), 287\left(\mathrm{M}^{+}-\mathrm{C}_{2} \mathrm{H}_{4} \mathrm{~N}_{2} \mathrm{O}, 12\right)$ and $275\left(8, \mathrm{M}^{+}-\mathrm{C}_{2} \mathrm{H}_{2} \mathrm{~N}_{3} \mathrm{O}\right)$; Anal. Calc. for $\mathrm{C}_{20} \mathrm{H}_{17} \mathrm{~N}_{5} \mathrm{O}_{2}$ (359.38): C, 66.84; H, 4.77; N, 19.49\%, found: C, 66.80; H, 4.82; N, 19.50\%.

5-(p-Chlorophenyl)-7-formylhydrazino-2-phenylpyrazolo[1,5-c]pyrimidine (6d). Yield 69\%, $0.25 \mathrm{~g}$, mp 175-176 ${ }^{\circ} \mathrm{C}$; IR $\left(v_{\max }, \mathrm{cm}^{-1}\right): 3315(\mathrm{NH}), 1700(\mathrm{C}=\mathrm{O}), 1615$ (pyrazole ring $\left.\mathrm{C}=\mathrm{N}\right), 1575$ (pyrimidine ring $\mathrm{C}=\mathrm{N})$, and $1450(\mathrm{C}=\mathrm{C}) ;{ }^{1} \mathrm{H} \mathrm{NMR}\left(\mathrm{CDCl}_{3}, \delta_{\mathrm{H}}, \mathrm{ppm}\right): 4.76(\mathrm{~s}, 2 \mathrm{H}$, exchangeable $2 \mathrm{NH})$, $6.72(\mathrm{~s}, 1 \mathrm{H}$, pyrazole-H), $7.28(\mathrm{~s}, 1 \mathrm{H}$, pyrimidine-H), 7.41-7.46 (m, 5H, aromatic-H), 7.95-7.99 (m, $4 \mathrm{H}$, aromatic- $\mathrm{H})$ and $8.00(\mathrm{~s}, 1 \mathrm{H}, \underline{\mathrm{CHO}})$; MS, $m / z(\%)=361\left(\mathrm{M}^{+}-3,2,\right), 335\left(\mathrm{M}^{+}+1-\mathrm{CO}, 19\right), 320$ 
$\left(\mathrm{M}^{+}-\mathrm{CHNO}, 100\right), 305\left(\mathrm{M}^{+}-\mathrm{CH}_{2} \mathrm{~N}_{2} \mathrm{O}, 47\right), 294\left(\mathrm{M}^{+}{ }^{+} \mathrm{C}_{2} \mathrm{HN}_{2} \mathrm{O}, 10\right), 269\left(\mathrm{M}^{+}-\mathrm{C}_{6} \mathrm{H}_{6} \mathrm{O}, 5\right)$ and 242 $\left(\mathrm{M}^{+}-\mathrm{C}_{7} \mathrm{H}_{7} \mathrm{NO}\right.$, 5); Anal. Calc. for $\mathrm{C}_{19} \mathrm{H}_{14} \mathrm{ClN}_{5} \mathrm{O}$ (363.80): C, 62.73; H, 3.88; N, 19.25\%, found: C, $62.70 ; \mathrm{H}, 3.90 ; \mathrm{N}, 19.30 \%$.

\subsubsection{7-Acetylhydrazino-5-aryl-2-phenylpyrazolo[1,5-c]pyrimidines 7a-d}

5-Aryl-7-hydrazino-2-phenylpyrazolo[1,5-c]pyrimidines 1a-d (1mmol) and glacial acetic acid $(10 \mathrm{~mL})$ was heated at reflux for $3 \mathrm{~h}$. The reaction mixture was poured onto crushed ice and the product which separated was filtered, washed with water, dried and crystallized from EtOH to give the title compounds $\mathbf{7 a - d}$ as colorless needles.

7-Acetylhydrazino-2,5-diphenylpyrazolo[1,5-c]pyrimidine (7a). Yield 88\%, $0.30 \mathrm{~g}, \mathrm{mp} 217-218{ }^{\circ} \mathrm{C}$; IR $\left(v_{\max }, \mathrm{cm}^{-1}\right): 3150(\mathrm{NH}), 1661(\mathrm{C}=\mathrm{O}), 1628($ pyrazole ring $\mathrm{C}=\mathrm{N}), 1568$ (pyrimidine ring $\left.\mathrm{C}=\mathrm{N}\right)$, and $1459(\mathrm{C}=\mathrm{C}) ;{ }^{1} \mathrm{H}$ NMR $\left(\mathrm{CDCl}_{3}, \delta_{\mathrm{H}}, \mathrm{ppm}\right): 2.21\left(\mathrm{~s}, 3 \mathrm{H}, \mathrm{COCH}_{3}\right), 6.67(\mathrm{~s}, 1 \mathrm{H}$, pyrazole-H), $7.21(\mathrm{~s}, 1 \mathrm{H}$, pyrimidine- $\mathrm{H}), 7.30-7.51(\mathrm{~m}, 5 \mathrm{H}$, aromatic- $\mathrm{H}), 7.84-7.96(\mathrm{~m}, 5 \mathrm{H}$, aromatic- $\mathrm{H}), 8.01(\mathrm{~s}, 1 \mathrm{H}$, exchangeable $\mathrm{NH})$ and $8.48(\mathrm{~s}, 1 \mathrm{H}$, exchangeable $\mathrm{NHCO}) ; \mathrm{MS}, m / z(\%)=343\left(\mathrm{M}^{+}, 100\right), 328$ $\left(\mathrm{M}^{+}-\mathrm{CH}_{3}, 1\right), 301\left(\mathrm{M}^{+}{ }^{-}-\mathrm{COCH}_{2}, 59\right), 286\left(\mathrm{M}^{+}-\mathrm{NCOCH}_{3}, 18\right)$ and $243\left(\mathrm{M}^{+}-\mathrm{C}_{3} \mathrm{H}_{6} \mathrm{~N}_{3} \mathrm{O}, 11\right)$; Anal. Calc. for $\mathrm{C}_{20} \mathrm{H}_{17} \mathrm{~N}_{5} \mathrm{O}(343.38)$ : C, 69.96; H, 4.99; N, 20.40\%, found: C, 70.00; H, 5.02; N, 20.43\%.

7-Acetylhydrazino-2-phenyl-5-p-tolylpyrazolo[1,5-c]pyrimidine (7b). Yield 83\%, 0.30 g, mp 224-225 ${ }^{\circ} \mathrm{C}$; IR $\left(v_{\max }, \mathrm{cm}^{-1}\right)$ : $3252(\mathrm{NH}), 1659(\mathrm{C}=\mathrm{O}), 1618$ (pyrazole ring $\mathrm{C}=\mathrm{N}$ ), 1556 (pyrimidine ring $\mathrm{C}=\mathrm{N})$, and $1445(\mathrm{C}=\mathrm{C}) ;{ }^{1} \mathrm{H} \mathrm{NMR}\left(\mathrm{CDCl}_{3}, \delta_{\mathrm{H}}, \mathrm{ppm}\right): 2.23\left(\mathrm{~s}, 3 \mathrm{H}, \underline{\mathrm{CH}}_{3}\right), 2.36\left(\mathrm{~s}, 3 \mathrm{H}, \mathrm{COCH}_{3}\right), 6.70$ $(\mathrm{s}, 1 \mathrm{H}$, pyrazole-H), $7.19(\mathrm{~d}, 2 \mathrm{H}$, aromatic- $\mathrm{H}), 7.26(\mathrm{~s}, 1 \mathrm{H}$, pyrimidine- $\mathrm{H}), 7.36-7.48(\mathrm{~m}, 3 \mathrm{H}$, aromatic$\mathrm{H}), 7.79(\mathrm{~d}, 2 \mathrm{H}$, aromatic-H), $7.97(\mathrm{~d}, 2 \mathrm{H}$, aromatic-H), $8.14(\mathrm{~s}, 1 \mathrm{H}$, exchangeable $\mathrm{NH})$ and 8.31 $(\mathrm{s}, 1 \mathrm{H}$, exchangeable $\mathrm{NHCO}) ; \mathrm{MS}, m / z(\%)=358\left(\mathrm{M}^{+}+1,100\right), 315\left(\mathrm{M}^{+}-\mathrm{COCH}_{2}, 63\right), 300$ $\left(\mathrm{M}^{+}-\mathrm{NCOCH}_{3}, 19\right), 288\left(\mathrm{M}^{+}-\mathrm{C}_{3} \mathrm{H}_{3} \mathrm{NO}, 6\right)$ and $259\left(\mathrm{M}^{+}-\mathrm{C}_{3} \mathrm{H}_{4} \mathrm{~N}_{3} \mathrm{O}\right.$, 5); Anal. Calc. for $\mathrm{C}_{21} \mathrm{H}_{19} \mathrm{~N}_{5} \mathrm{O}$ (357.41): C, 70.57; H, 5.36; N, 19.59\%, found: C, 70.60; H, 5.32; N, 19.55\%.

7-Acetylhydrazino-5-p-methoxyphenyl-2-phenylpyrazolo[1,5-c]pyrimidine (7c). Yield 81\%, $0.30 \mathrm{~g}, \mathrm{mp}$ 193-194 ${ }^{\circ} \mathrm{C}$; IR $\left(v_{\max }, \mathrm{cm}^{-1}\right)$ : $3283(\mathrm{NH}), 1664(\mathrm{C}=\mathrm{O}), 1618$ (pyrazole ring $\mathrm{C}=\mathrm{N}$ ), 1564 (pyrimidine ring $\mathrm{C}=\mathrm{N})$, and $1448(\mathrm{C}=\mathrm{C}) ;{ }^{1} \mathrm{H} \mathrm{NMR}\left(\mathrm{CDCl}_{3}, \delta_{\mathrm{H}}, \mathrm{ppm}\right): 2.24\left(\mathrm{~s}, 3 \mathrm{H}, \mathrm{COCH}_{3}\right), 3.78\left(\mathrm{~s}, 3 \mathrm{H}, \underline{\mathrm{OCH}}_{3}\right)$, $6.63(\mathrm{~s}, 1 \mathrm{H}$, pyrazole-H), $6.88(\mathrm{~d}, 2 \mathrm{H}$, aromatic- $\mathrm{H}), 7.07(\mathrm{~s}, 1 \mathrm{H}$, pyrimidine- $\mathrm{H}), 7.36-7.50(\mathrm{~m}, 3 \mathrm{H}$, aromatic-H), $7.76(\mathrm{~d}, 2 \mathrm{H}$, aromatic- $\mathrm{H}), 7.91(\mathrm{~d}, 2 \mathrm{H}$, aromatic- $\mathrm{H}), 8.13(\mathrm{~s}, 1 \mathrm{H}$, exchangeable $\mathrm{NH})$ and $8.91\left(\mathrm{~s}, 1 \mathrm{H}\right.$, exchangeable NHCO); MS, $m / z(\%)=374\left(\mathrm{M}^{+}+1,100\right), 331\left(\mathrm{M}^{+}-\mathrm{COCH}_{2}, 38\right), 316$ $\left(\mathrm{M}^{+}-\mathrm{NCOCH}_{3}, 16\right), 302\left(\mathrm{M}^{+}-\mathrm{NNCOCH}_{3}, 56\right), 287\left(\mathrm{M}^{+}-\mathrm{C}_{3} \mathrm{H}_{6} \mathrm{~N}_{2} \mathrm{O}, 8\right)$ and $259\left(\mathrm{M}^{+}-\mathrm{C}_{4} \mathrm{H}_{8} \mathrm{~N}_{3} \mathrm{O}, 19\right)$; Anal. Calc. for $\mathrm{C}_{21} \mathrm{H}_{19} \mathrm{~N}_{5} \mathrm{O}_{2}$ (373.41): C, 67.55; H, 5.13; N, 18.76\%, found: C, 67.58; H, 5.16; N, 18.80\%.

7-Acetylhydrazino-5-p-Chlorophenyl-2-phenylpyrazolo[1,5-c]pyrimidine (7d). Yield 79\%, $0.30 \mathrm{~g}, \mathrm{mp}$ 246-247 ${ }^{\circ} \mathrm{C}$; IR $\left(v_{\max }, \mathrm{cm}^{-1}\right)$ : $3306(\mathrm{NH}), 1664(\mathrm{C}=\mathrm{O}), 1613$ (pyrazole ring $\mathrm{C}=\mathrm{N}$ ), 1572 (pyrimidine ring $\mathrm{C}=\mathrm{N})$, and $1447(\mathrm{C}=\mathrm{C}) ;{ }^{1} \mathrm{H}$ NMR (DMSO- $\left.d_{6}, \delta_{\mathrm{H}}, \mathrm{ppm}\right): 2.04\left(\mathrm{~s}, 3 \mathrm{H}, \mathrm{COCH}_{3}\right), 7.08(\mathrm{~s}, 1 \mathrm{H}$, pyrazole-H), 7.67 (s, 1H, pyrimidine-H), 7.41-7.52 (m, 5H, aromatic-H), 8.09 (d, 2H, aromatic-H), $9.81(\mathrm{~s}, 1 \mathrm{H}$, exchangeable $\mathrm{NH})$ and $10.14(\mathrm{~s}, 1 \mathrm{H}$, exchangeable $\mathrm{NHCO}) ; \mathrm{MS}, m / z(\%)=382\left(\mathrm{M}^{+}+4\right.$, 
1), $381\left(\mathrm{M}^{+}+3,4\right), 379\left(\mathrm{M}^{++}+1,44\right), 378\left(\mathrm{M}^{+}, 100\right), 336\left(\mathrm{M}^{+}-\mathrm{COCH}_{2}, 65\right), 320\left(\mathrm{M}^{+}-\mathrm{NHCOCH}_{3}, 16\right)$, $306\left(\mathrm{M}^{+}-\mathrm{NNHCOCH}_{3}, 77\right)$ and $270\left(\mathrm{M}^{+}-\mathrm{C}_{2} \mathrm{H}_{5} \mathrm{ClN}_{2} \mathrm{O}, 10\right)$; Anal. Calc. for $\mathrm{C}_{20} \mathrm{H}_{16} \mathrm{ClN}_{5} \mathrm{O}$ (377.83): C, $63.58 ; \mathrm{H}, 4.27$; N, 18.54\%, found: $\mathrm{C}, 63.61 ; \mathrm{H}, 4.26 ; \mathrm{N}, 18.60 \%$.

\subsubsection{7-Acetylhydrazino-5-aryl-3,4-dibromo-2--phenylpyrazolo[1,5-c]pyrimidines 8a-d}

A solution of bromine $(0.12 \mathrm{~mL}, 2.4 \mathrm{mmol})$ in acetic acid $(10 \mathrm{~mL})$ was gradually added to a suspension of 7-acetylhydrazino-5-aryl-2-phenylpyrazolo[1,5-c]pyrimidines 7a-d (1 mmol) in acetic acid $(10 \mathrm{~mL})$ with stirring for $3 \mathrm{~h}$ at room temperature. The precipitated 7-acetylhydrazino-5-aryl-3,4dibromo-2-phenylpyrazolo[1,5-c]pyrimidines 8a-d were filtered, washed with water, dried and crystallized from EtOH as colorless needles.

7-Acetylhydrazino-3,4-dibromo-2,5-diphenylpyrazolo[1,5-c]pyrimidine (8a). Yield 80\%, $0.40 \mathrm{~g}, \mathrm{mp}$ 229-230 ${ }^{\circ} \mathrm{C}$; IR $\left(v_{\max }, \mathrm{cm}^{-1}\right)$ : $3451(\mathrm{NH}), 1657(\mathrm{C}=\mathrm{O}), 1644$ (pyrazole ring $\mathrm{C}=\mathrm{N}$ ), 1560 (pyrimidine ring $\mathrm{C}=\mathrm{N})$, and $1439(\mathrm{C}=\mathrm{C}) ;{ }^{1} \mathrm{H} \mathrm{NMR}\left(\mathrm{CDCl}_{3}, \delta_{\mathrm{H}}, \mathrm{ppm}\right): 2.09\left(\mathrm{~s}, 3 \mathrm{H}, \mathrm{COCH}_{3}\right), 7.44-7.53(\mathrm{~m}, 6 \mathrm{H}$, aromatic-H), $7.63(\mathrm{~d}, 2 \mathrm{H}$, aromatic- $\mathrm{H}), 7.94(\mathrm{~d}, 2 \mathrm{H}$, aromatic-H) and $8.01(\mathrm{~s}, 2 \mathrm{H}$, exchangeable $\mathrm{NH}$ and exchangeable NHCO); MS, $m / z(\%)=505\left(\mathrm{M}^{+}+4,4\right), 503\left(\mathrm{M}^{+}+2,38\right), 501\left(\mathrm{M}^{+}, 100\right), 459\left(\mathrm{M}^{+}{ }_{-}\right.$ $\left.\mathrm{COCH}_{2}, 80\right), 444\left(\mathrm{M}^{+}-\mathrm{NCOCH}_{3}, 14\right), 429\left(\mathrm{M}^{+}-\mathrm{NNHCOCH}_{3}, 40\right), 423\left(\mathrm{M}^{+}+2-\mathrm{Br}, 21\right), 421\left(\mathrm{M}^{+}-\mathrm{Br}\right.$, 19) and $341\left(\mathrm{M}^{+}-\mathrm{Br} 2,2\right)$; Anal. Calc. for $\mathrm{C}_{20} \mathrm{H}_{15} \mathrm{Br}_{2} \mathrm{~N}_{5} \mathrm{O}$ (501.17): C, 47.93; H, 3.02; N, 13.97\%, found: C, 47.95; H, 3.06; N, 14.00\%.

7-Acetylhydrazino-3,4-dibromo-2-phenyl-5-p-tolylpyrazolo[1,5-c]pyrimidine $(\mathbf{8 b})$. Yield 77\%, $0.40 \mathrm{~g}$, mp 212-213 ${ }^{\circ} \mathrm{C}$; IR $\left(v_{\max }, \mathrm{cm}^{-1}\right): 3280(\mathrm{NH}), 1664(\mathrm{C}=\mathrm{O}), 1618$ (pyrazole ring $\left.\mathrm{C}=\mathrm{N}\right), 1562$ (pyrimidine ring $\mathrm{C}=\mathrm{N})$, and $1439(\mathrm{C}=\mathrm{C}) ;{ }^{1} \mathrm{H} \mathrm{NMR}\left(\mathrm{CDCl}_{3}, \delta_{\mathrm{H}}, \mathrm{ppm}\right): 2.10\left(\mathrm{~s}, 3 \mathrm{H}, \underline{\mathrm{CH}}_{3}\right), 2.40(\mathrm{~s}, 3 \mathrm{H}$, $\left.\mathrm{COCH}_{3}\right), 7.46-7.55(\mathrm{~m}, 5 \mathrm{H}$, aromatic-H), $7.86(\mathrm{~d}, 2 \mathrm{H}$, aromatic- $\mathrm{H}), 7.93(\mathrm{~d}, 2 \mathrm{H}$, aromatic- $\mathrm{H}), 8.04(\mathrm{~s}$, $1 \mathrm{H}$, exchangeable $\mathrm{NH})$ and $8.06(\mathrm{~s}, 1 \mathrm{H}$, exchangeable $\mathrm{NHCO}) ; \mathrm{MS}, m / z(\%)=519\left(\mathrm{M}^{+}+4,1\right), 517$ $\left(\mathrm{M}^{+}+2,15\right), 515\left(\mathrm{M}^{+}, 33\right), 473\left(\mathrm{M}^{+}-\mathrm{COCH}_{2}, 18\right), 458\left(\mathrm{M}^{+}-\mathrm{NCOCH}_{3}, 2\right), 444\left(\mathrm{M}^{+}-\mathrm{NNCOCH}_{3}, 7\right)$, $442\left(\mathrm{M}^{+}-\mathrm{NHNHCOCH}_{3}, 5\right), 437\left(\mathrm{M}^{+}+2-\mathrm{Br}, 100\right), 435\left(\mathrm{M}^{+}-\mathrm{Br}, 84\right)$ and $355\left(\mathrm{M}^{+}-\mathrm{Br}_{2}, 1\right)$; Anal. Calc. for $\mathrm{C}_{21} \mathrm{H}_{17} \mathrm{Br}_{2} \mathrm{~N}_{5} \mathrm{O}$ (515.20): C, 48.96; H, 3.33; N, 13.59\%, found: C, 49.00; H, 3.36; N, 13.60\%.

7-Acetylhydrazino-3,4-dibromo-5-(p-methoxyphenyl)-2-phenylpyrazolo[1,5-c]-pyrimidine (8c). Yield 75\%, $0.40 \mathrm{~g}, \mathrm{mp} 182-183{ }^{\circ} \mathrm{C}$; IR $\left(\mathrm{v}_{\max }, \mathrm{cm}^{-1}\right): 3269(\mathrm{NH}), 1668(\mathrm{C}=\mathrm{O}), 1612$ (pyrazole ring $\left.\mathrm{C}=\mathrm{N}\right)$, 1535 (pyrimidine ring $\mathrm{C}=\mathrm{N})$, and $1443(\mathrm{C}=\mathrm{C}) ;{ }^{1} \mathrm{H} \mathrm{NMR}\left(\mathrm{CDCl}_{3}, \delta_{\mathrm{H}}, \mathrm{ppm}\right): 2.47\left(\mathrm{~s}, 3 \mathrm{H}, \mathrm{COCH}_{3}\right), 3.79$ $\left(\mathrm{s}, 3 \mathrm{H}, \mathrm{OCH}_{3}\right), 7.48-7.62(\mathrm{~m}, 3 \mathrm{H}$, aromatic- $\mathrm{H}), 7.91(\mathrm{~d}, 2 \mathrm{H}$, aromatic- $\mathrm{H}), 8.04(\mathrm{~d}, 2 \mathrm{H}$, aromatic- $\mathrm{H})$, $8.08(\mathrm{~d}, 2 \mathrm{H}$, aromatic-H), $10.05(\mathrm{~s}, 1 \mathrm{H}$, exchangeable $\mathrm{NH})$ and $10.10(\mathrm{~s}, 1 \mathrm{H}$, exchangeable $\mathrm{NHCO})$; MS, $m / z(\%)=535\left(\mathrm{M}^{+}+4,1\right), 533\left(\mathrm{M}^{+}+2,6\right), 531\left(\mathrm{M}^{+}, 10\right), 489\left(\mathrm{M}^{+}-\mathrm{COCH}_{2}, 6\right), 475\left(\mathrm{M}^{+}-\mathrm{NCOCH}_{2}\right.$, 13), $459\left(\mathrm{M}^{+}{ }^{-} \mathrm{NNHCOCH}_{3}, 23\right), 453\left(\mathrm{M}^{+}+2-\mathrm{Br}, 73\right), 451\left(\mathrm{M}^{+}-\mathrm{Br}, 61\right)$ and $370\left(\mathrm{M}^{+}+1-\mathrm{Br}_{2}, 1\right)$; Anal. Calc. for $\mathrm{C}_{21} \mathrm{H}_{17} \mathrm{Br}_{2} \mathrm{~N}_{5} \mathrm{O}_{2}$ (531.20): C, 47.48; H, 3.23; N, 13.18\%, found: C, 47.50; H, 3.26; N, 13.20\%.

7-Acetylhydrazino-5-(p-chlorophenyl)-3,4-dibromo-2-phenylpyrazolo[1,5-c]-pyrimidine (8d). Yield 74\%, $0.40 \mathrm{~g}, \mathrm{mp} 226-227^{\circ} \mathrm{C}$; IR $\left(v_{\max }, \mathrm{cm}^{-1}\right): 3267(\mathrm{NH}), 1664(\mathrm{C}=\mathrm{O}), 1620$ (pyrazole ring $\left.\mathrm{C}=\mathrm{N}\right)$,

1570 (pyrimidine ring $\mathrm{C}=\mathrm{N}$ ), and $1437(\mathrm{C}=\mathrm{C}) ;{ }^{1} \mathrm{H}$ NMR (DMSO- $\left.d_{6}, \delta_{\mathrm{H}}, \mathrm{ppm}\right): 2.47\left(\mathrm{~s}, 3 \mathrm{H}, \mathrm{COCH}_{3}\right)$, 7.49-7.57 (m, 5H, aromatic-H), $8.04(\mathrm{~d}, 2 \mathrm{H}$, aromatic-H), $8.15(\mathrm{~d}, 2 \mathrm{H}$, aromatic-H), $10.06(\mathrm{~s}, 1 \mathrm{H}$, 
exchangeable $\mathrm{NH}$ ) and $10.17\left(\mathrm{~s}, 1 \mathrm{H}\right.$, exchangeable NHCO); $\mathrm{MS}, m / z(\%)=538\left(\mathrm{M}^{+}+2,2\right), 535$ $\left(\mathrm{M}^{+}-1,4\right), 493\left(\mathrm{M}^{+}-\mathrm{COCH}_{3}, 4\right), 463\left(\mathrm{M}^{+}{ }^{-} \mathrm{NHNHCOCH}_{3}, 3\right), 458\left(\mathrm{M}^{+}+2-\mathrm{Br}, 20\right), 456\left(\mathrm{M}^{+}-\mathrm{Br}, 100\right)$ and $376\left(\mathrm{M}^{+}-\mathrm{Br}_{2}, 1\right)$; Anal. Calc. for $\mathrm{C}_{20} \mathrm{H}_{14} \mathrm{Br}_{2} \mathrm{ClN}_{5} \mathrm{O}$ (535.62): $\mathrm{C}, 44.85 ; \mathrm{H}, 2.63 ; \mathrm{N}, 13.08 \%$, found: C, 44.81; H, 2.60; N, 13.11\%.

\subsubsection{7-Acetylhydrazino-5-aryl-3-iodo-2--phenylpyrazolo[1,5-c]pyrimidines 9a-d}

A solution of iodine monochloride $(0.2 \mathrm{~g}, 1.2 \mathrm{mmol})$ in acetic acid $(10 \mathrm{~mL})$ was gradually added to a suspension of 7-acetylhydrazino-5-aryl-2-phenylpyrazolo[1,5-c]pyrimidines 7a-d (1 mmol) in acetic acid $(10 \mathrm{~mL})$ with stirring for $3 \mathrm{~h}$ at room temperature. The reaction mixture was then poured onto crushed ice and the precipitated 7-acetylhydrazino-5-aryl-3-iodo-2-phenylpyrazolo[1,5-c]pyrimidines 9a-d were filtered, washed with water, dried and crystallized from EtOH as colorless needles.

7-Acetylhydrazino-3-iodo-2,5-diphenylpyrazolo[1,5-c]pyrimidine (9a). Yield 85\%, $0.40 \mathrm{~g}, \mathrm{mp} 229$ $230{ }^{\circ} \mathrm{C}$; IR $\left(v_{\max }, \mathrm{cm}^{-1}\right): 3433(\mathrm{NH}), 1680(\mathrm{C}=\mathrm{O}), 1618$ (pyrazole ring $\mathrm{C}=\mathrm{N}$ ), 1549 (pyrimidine ring $\mathrm{C}=\mathrm{N})$, and $1438(\mathrm{C}=\mathrm{C}) ;{ }^{1} \mathrm{H} \mathrm{NMR}\left(\mathrm{DMSO}-d_{6}, \delta_{\mathrm{H}}, \mathrm{ppm}\right): 2.05\left(\mathrm{~s}, 3 \mathrm{H}, \mathrm{CO} \underline{\mathrm{CH}}_{3}\right), 7.29-7.80(\mathrm{~m}, 10 \mathrm{H}$, aromatic-H), $7.48(\mathrm{~s}, 1 \mathrm{H}$, pyrimidine-H), and $8.06(\mathrm{~s}, 2 \mathrm{H}$, exchangeable NHCO and exchangeable $\mathrm{NH})$; MS, $m / z(\%)=469\left(\mathrm{M}^{+}, 2\right), 451\left(\mathrm{M}^{+}-\mathrm{H}_{2} \mathrm{O}, 4\right), 437\left(\mathrm{M}^{+}-\mathrm{CH}_{4} \mathrm{O}, 2\right), 343\left(\mathrm{M}^{+}+1-\mathrm{I}, 5\right), 300\left(\mathrm{M}^{+}-\right.$ $\left.\mathrm{I}-\mathrm{CH}_{2} \mathrm{CO}, 32\right)$ and $385\left(\mathrm{M}^{+}-\mathrm{CH}_{3} \mathrm{INO}, 100\right)$; Anal. Calc. for $\mathrm{C}_{20} \mathrm{H}_{16} \mathrm{IN}_{5} \mathrm{O}$ (469.28): C, 51.19; H, 3.44; N, $14.92 \%$, found: C, $51.20 ; \mathrm{H}, 3.40 ; \mathrm{N}, 14.88 \%$.

7-Acetylhydrazino-3-iodo-2-phenyl-5-p-tolylpyrazolo[1,5-c]pyrimidine (9b). Yield 83\%, $0.40 \mathrm{~g}, \mathrm{mp}$ 204-205 ${ }^{\circ} \mathrm{C}$; IR $\left(v_{\max }, \mathrm{cm}^{-1}\right)$ : $3273(\mathrm{NH}), 1661(\mathrm{C}=\mathrm{O}), 1610$ (pyrazole ring $\mathrm{C}=\mathrm{N}$ ), 1564 (pyrimidine ring $\mathrm{C}=\mathrm{N})$, and $1433(\mathrm{C}=\mathrm{C})$; ${ }^{1} \mathrm{H}$ NMR $\left(\mathrm{CDCl}_{3}, \delta_{\mathrm{H}}, \mathrm{ppm}\right): 2.26\left(\mathrm{~s}, 3 \mathrm{H}, \mathrm{CH}_{3}\right), 2.42\left(\mathrm{~s}, 3 \mathrm{H}, \mathrm{COCH}_{3}\right), 7.18$ $(\mathrm{s}, 1 \mathrm{H}$, pyrimidine- $\mathrm{H}), 7.28-7.30(\mathrm{~m}, 3 \mathrm{H}$, aromatic- $\mathrm{H}), 7.49-7.52(\mathrm{~m}, 4 \mathrm{H}$, aromatic- $\mathrm{H}), 7.84(\mathrm{~d}, 2 \mathrm{H}$, aromatic-H), $7.97(\mathrm{~s}, 1 \mathrm{H}$, exchangeable $\mathrm{NH})$, and $7.99(\mathrm{~s}, 1 \mathrm{H}$, exchangeable NHCO); $\mathrm{MS}, m / z(\%)=$ $484\left(\mathrm{M}^{++}+1,83\right), 441\left(\mathrm{M}^{+}-\mathrm{COCH}_{2}, 34\right), 426\left(\mathrm{M}^{+}-\mathrm{NCOCH}_{3}, 12\right), 412\left(\mathrm{M}^{+}-\mathrm{NNCOCH}_{3}, 12\right), 410\left(\mathrm{M}^{+}{ }_{-}\right.$ $\left.\mathrm{NHNHCOCH}_{3}, 1\right)$ and $357\left(\mathrm{M}^{+}+1-\mathrm{I}, 16\right)$; Anal. Calc. for $\mathrm{C}_{21} \mathrm{H}_{18} \mathrm{IN}_{5} \mathrm{O}$ (483.30): C, 52.19; H, 3.75; N, $14.49 \%$, found: C, 52.50; H, 3.79; N, 14.52\%.

7-Acetylhydrazino-3-iodo-5-(p-methoxyphenyl)-2-phenylpyrazolo[1,5-c]-pyrimidine (9c). Yield 80\%, $0.40 \mathrm{~g}, \mathrm{mp} 124-125{ }^{\circ} \mathrm{C}$; IR $\left(\mathrm{v}_{\max }, \mathrm{cm}^{-1}\right): 3290(\mathrm{NH}), 1711(\mathrm{C}=\mathrm{O}), 1610$ (pyrazole ring $\left.\mathrm{C}=\mathrm{N}\right), 1560$ (pyrimidine ring $\mathrm{C}=\mathrm{N})$, and $1448(\mathrm{C}=\mathrm{C}) ;{ }^{1} \mathrm{H} \mathrm{NMR}\left(\mathrm{CDCl}_{3}, \delta_{\mathrm{H}}, \mathrm{ppm}\right): 2.16\left(\mathrm{~s}, 3 \mathrm{H}, \mathrm{COCH}_{3}\right), 3.87$ (s, $\left.3 \mathrm{H}, \mathrm{OCH}_{3}\right), 6.99-7.49(\mathrm{~m}, 7 \mathrm{H}$, aromatic-H), $7.69(\mathrm{~s}, 1 \mathrm{H}$, pyrimidine- $\mathrm{H}), 8.01(\mathrm{~d}, 2 \mathrm{H}$, aromatic- $\mathrm{H})$ and $9.28(\mathrm{~s}, 2 \mathrm{H}$, exchangeable $\mathrm{NH}$ and exchangeable $\mathrm{NHCO})$; $\mathrm{MS}, m / z(\%)=484\left(\mathrm{M}^{+}{ }^{-} \mathrm{CH}_{3}, 1\right), 343\left(\mathrm{M}^{+}{ }_{-}\right.$ $\left.\mathrm{NCOCH}_{2}, 3\right), 373\left(\mathrm{M}^{+}+1-\mathrm{I}, 6\right)$ and $372\left(\mathrm{M}^{+}-\mathrm{I}, 1\right)$; Anal. Calc. for $\mathrm{C}_{21} \mathrm{H}_{18} \mathrm{IN}_{5} \mathrm{O}_{2}$ (499.30): C, 50.52; $\mathrm{H}$, 3.63 ; N, 14.03\%. Found: C, 50.55; H, 3.59; N, 14.06\%.

7-Acetylhydrazino-5-(p-chlorophenyl)-3-iodo-2-phenylpyrazolo[1,5-c]pyrimidine (9d). Yield 80\%, $0.40 \mathrm{~g}, \mathrm{mp} 207-208{ }^{\circ} \mathrm{C}$; IR $\left(\mathrm{v}_{\max }, \mathrm{cm}^{-1}\right): 3273(\mathrm{NH}), 1662(\mathrm{C}=\mathrm{O}), 1616$ (pyrazole ring $\left.\mathrm{C}=\mathrm{N}\right), 1566$ (pyrimidine ring $\mathrm{C}=\mathrm{N})$, and $1439(\mathrm{C}=\mathrm{C}) ;{ }^{1} \mathrm{H}$ NMR (DMSO- $\left.d_{6}, \delta_{\mathrm{H}}, \mathrm{ppm}\right): 2.01\left(\mathrm{~s}, 3 \mathrm{H}, \mathrm{COC} \underline{\mathrm{H}}_{3}\right), 7.14$ (d, 2H, aromatic-H), $7.40(\mathrm{~s}, 1 \mathrm{H}$, pyrimidine-H), 7.49-7.54 (m, 3H, aromatic- $\mathrm{H}), 7.68(\mathrm{~d}, 1 \mathrm{H}$, aromatic-H), $7.80(\mathrm{~d}, 1 \mathrm{H}$, aromatic-H), $7.98(\mathrm{~d}, 2 \mathrm{H}$, aromatic-H),. $10.00(\mathrm{~s}, 1 \mathrm{H}$, exchangeable $\mathrm{NH})$, and 
$10.16\left(\mathrm{~s}, 1 \mathrm{H}\right.$, exchangeable NHCO); MS, $m / z(\%)=506\left(\mathrm{M}^{++}+2,45\right), 504\left(\mathrm{M}^{+}, 100\right), 463\left(\mathrm{M}^{++}+2-\right.$ $\left.\mathrm{COCH}_{3}, 22\right), 461\left(\mathrm{M}^{+}-\mathrm{COCH}_{3}, 57\right), 446\left(\mathrm{M}^{+}-\mathrm{NHCOCH}_{3}, 15\right), 432\left(\mathrm{M}^{+}-\mathrm{NNHCOCH}_{3}, 17\right), 379$ $\left(\mathrm{M}^{+}+2-\mathrm{I}, 10\right)$ and $377\left(\mathrm{M}^{+}-\mathrm{I}, 24\right)$; Anal. Calc. for $\mathrm{C}_{20} \mathrm{H}_{15} \mathrm{ClIN}_{5} \mathrm{O}$ (503.72): C, 47.69; H, 3.00; N, $13.90 \%$, found: C, 47.72; H, 2.99; N, 13.92\%.

\subsubsection{7-Acetylhydrazino-5-aryl-3-nitro-2-phenylpyrazolo[1,5-c]pyrimidines 10a-d}

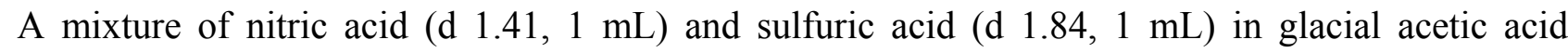
$(10 \mathrm{~mL})$ was gradually added to a suspension of 7-acetylhydrazino-5-aryl-2-phenylpyrazolo[1,5c]pyrimidines 7a-d $(1 \mathrm{mmol})$ in glacial acetic acid $(10 \mathrm{~mL})$ with stirring for $3 \mathrm{~h}$ at room temperature. The reaction mixture was then poured onto cold water with stirring and the yellow precipitated solid were filtered, washed with cold water, dried and crystallized from EtOH to give the title compounds

10a-d as yellow needles.

7-Acetylhydrazino-3-nitro-2,5-diphenylpyrazolo[1,5-c]pyrimidine (10a). Yield 77\%, $0.30 \mathrm{~g}, \mathrm{mp} 191-$ $192{ }^{\circ} \mathrm{C}$; IR $\left(v_{\max }, \mathrm{cm}^{-1}\right): 3436(\mathrm{NH}), 1743(\mathrm{C}=\mathrm{O}), 1633$ (pyrazole ring $\mathrm{C}=\mathrm{N}$ ), 1551 (pyrimidine ring $\mathrm{C}=\mathrm{N})$, and $1462(\mathrm{C}=\mathrm{C}) ;{ }^{1} \mathrm{H} \mathrm{NMR}\left(\mathrm{CDCl}_{3}, \delta_{\mathrm{H}}, \mathrm{ppm}\right): 1.90\left(\mathrm{~s}, 3 \mathrm{H}, \mathrm{COCH}_{3}\right), 7.37(\mathrm{~s}, 1 \mathrm{H}$, pyrimidine-H), 7.44-7.68 (m, 6H, aromatic- $\mathrm{H}), 7.97(\mathrm{~d}, 2 \mathrm{H}$, aromatic- $\mathrm{H}), 8.03(\mathrm{~d}, 2 \mathrm{H}$, aromatic- $\mathrm{H}), 8.46(\mathrm{~s}, 1 \mathrm{H}$, exchangeable $\mathrm{NH})$, and $9.26(\mathrm{~s}, 1 \mathrm{H}$, exchangeable $\mathrm{NHCO})$; $\mathrm{MS}, m / z(\%)=389\left(\mathrm{M}^{+}+1,4\right), 360\left(\mathrm{M}^{+}{ }_{-}\right.$ $\mathrm{CO}, \quad 7), 346\left(\mathrm{M}^{+}-\mathrm{COCH}_{2}, \quad 2\right), 332\left(\mathrm{M}^{+}-\mathrm{NCOCH}_{2}, 100\right), 316\left(\mathrm{M}^{+}-\mathrm{NNHCOCH}_{3}, 4\right), 302$ $\left(\mathrm{M}^{+}-\mathrm{C}_{3} \mathrm{H}_{6} \mathrm{~N}_{2} \mathrm{O}, 17\right), 286\left(\mathrm{M}^{+}-\mathrm{C}_{2} \mathrm{H}_{2} \mathrm{~N}_{2} \mathrm{O}_{3}, 10\right)$ and $258\left(\mathrm{M}^{+}-\mathrm{C}_{3} \mathrm{H}_{4} \mathrm{~N}_{3} \mathrm{O}_{3}, 25\right)$; Anal. Calc. for $\mathrm{C}_{20} \mathrm{H}_{16} \mathrm{~N}_{6} \mathrm{O}_{3}$ (388.38): C, 61.85; H, 4.15; N, 21.64\%, found: C, 61.89; H, 4.12; N, 21.60\%.

7-Acetylhydrazino-3-nitro-2-phenyl-5-p-tolylpyrazolo[1,5-c]pyrimidine (10b). Yield 75\%, $0.30 \mathrm{~g}, \mathrm{mp}$ 194-195 ${ }^{\circ} \mathrm{C}$; IR $\left(v_{\max }, \mathrm{cm}^{-1}\right)$ : $3371(\mathrm{NH}), 1745(\mathrm{C}=\mathrm{O}), 1601$ (pyrazole ring $\mathrm{C}=\mathrm{N}$ ), 1533 (pyrimidine ring $\mathrm{C}=\mathrm{N})$, and $1443(\mathrm{C}=\mathrm{C}) ;{ }^{1} \mathrm{H} \mathrm{NMR}\left(\mathrm{CDCl}_{3}, \delta_{\mathrm{H}}, \mathrm{ppm}\right): 1.96\left(\mathrm{~s}, 3 \mathrm{H}, \underline{\mathrm{CH}}_{3}\right), 2.44\left(\mathrm{~s}, 3 \mathrm{H}, \mathrm{COCH}_{3}\right)$, 7.30-7.71 (m, 10H, aromatic- $\mathrm{H}$ and pyrimidine- $\mathrm{H})$ and $8.44(\mathrm{~s}, 2 \mathrm{H}$, exchangeable $\mathrm{NH}$ and exchangeable NHCO); MS, $m / z(\%)=403\left(\mathrm{M}^{++}+1,2\right), 402\left(\mathrm{M}^{+}, 3\right), 401\left(\mathrm{M}^{+}-1,16\right), 374\left(\mathrm{M}^{+}-\mathrm{CO}, 8\right)$ and $346\left(\mathrm{M}^{+}-\mathrm{C}_{2} \mathrm{H}_{2} \mathrm{NO}, 6\right)$; Anal. Calc. for $\mathrm{C}_{21} \mathrm{H}_{18} \mathrm{~N}_{6} \mathrm{O}_{3}$ (402.41): C, 62.68; H, 4.51; N, 20.88\%, found: C, 62.71; H, 4.50; N, 20.91\%.

7-Acetylhydrazino-5-(p-methoxyphenyl)-3-nitro-2-phenylpyrazolo[1,5-c]-pyrimidine (10c). Yield 71\%, $0.30 \mathrm{~g}, \mathrm{mp} 132-133{ }^{\circ} \mathrm{C}$; IR $\left(v_{\max }, \mathrm{cm}^{-1}\right): 3464(\mathrm{NH}), 1747(\mathrm{C}=\mathrm{O}), 1696$ (pyrazole ring $\left.\mathrm{C}=\mathrm{N}\right), 1531$ (pyrimidine ring $\mathrm{C}=\mathrm{N})$, and $1454(\mathrm{C}=\mathrm{C}) ;{ }^{1} \mathrm{H} \mathrm{NMR}\left(\mathrm{CDCl}_{3}, \delta_{\mathrm{H}}, \mathrm{ppm}\right): 1.81\left(\mathrm{~s}, 3 \mathrm{H}, \mathrm{COCH}_{3}\right), 3.89$ (s, $\left.3 \mathrm{H} \mathrm{OCH}_{3}\right), 7.00-7.71(\mathrm{~m}, 10 \mathrm{H}$, aromatic-H and pyrimidine- $\mathrm{H})$ and $8.43(\mathrm{~s}, 2 \mathrm{H}$, exchangeable $\mathrm{NH}$ and exchangeable NHCO); MS, $m / z(\%)=420\left(\mathrm{M}^{+}+2,1\right), 358\left(\mathrm{M}^{+}{ }^{+} \mathrm{C}_{2} \mathrm{H}_{6} \mathrm{NO}, 1\right), 334\left(\mathrm{M}^{+}-\mathrm{C}_{3} \mathrm{H}_{4} \mathrm{~N}_{2} \mathrm{O}, 1\right)$ and $300\left(\mathrm{M}^{+}-\mathrm{C}_{2} \mathrm{H}_{4} \mathrm{~N}_{3} \mathrm{O}_{3}\right.$, 3); Anal. Calc. for $\mathrm{C}_{21} \mathrm{H}_{18} \mathrm{~N}_{6} \mathrm{O}_{4}$ (418.41): C, 60.28; H, 4.34; N, 20.09\%, found: $\mathrm{C}, 60.31 ; \mathrm{H}, 4.32 ; \mathrm{N}, 20.13 \%$.

7-Acetylhydrazino-5-(p-chlorophenyl)-3-nitro-2-phenylpyrazolo[1,5-c]-pyrimidine (10d). Yield 71\%, $0.30 \mathrm{~g}, \mathrm{mp} 174-175{ }^{\circ} \mathrm{C}$; IR $\left(\mathrm{v}_{\max }, \mathrm{cm}^{-1}\right): 3371(\mathrm{NH}), 1749(\mathrm{C}=\mathrm{O}), 1597$ (pyrazole ring $\left.\mathrm{C}=\mathrm{N}\right), 1533$ (pyrimidine ring $\mathrm{C}=\mathrm{N})$, and $1443(\mathrm{C}=\mathrm{C}) ;{ }^{1} \mathrm{H} \mathrm{NMR}\left(\mathrm{CDCl}_{3}, \delta_{\mathrm{H}}, \mathrm{ppm}\right): 2.13\left(\mathrm{~s}, 3 \mathrm{H}, \mathrm{COCH}_{3}\right), 7.34-7.73$ (m, 10H, aromatic-H and pyrimidine-H) and 8.45 (s, 2H, exchangeable $\mathrm{NH}$ and exchangeable NHCO); 
MS, $m / z(\%)=423\left(\mathrm{M}^{+}, 1\right), 381\left(\mathrm{M}^{+}-\mathrm{COCH}_{2}, 1\right), 366\left(\mathrm{M}^{+}-\mathrm{NCOCH}_{3}, 1\right), 324\left(\mathrm{M}^{+}-\mathrm{C}_{3} \mathrm{H}_{5} \mathrm{~N}_{3} \mathrm{O}, 1\right)$ and $300\left(\mathrm{M}^{+}-\mathrm{C}_{6} \mathrm{H}_{5} \mathrm{NO}_{2}, 2\right)$; Anal. Calc. for $\mathrm{C}_{20} \mathrm{H}_{15} \mathrm{ClN}_{6} \mathrm{O}_{3}$ (422.82): C, 56.81; H, 3.58; N, 19.88\%, found: C, 56.77; H, 3.61; N, $19.85 \%$.

\subsubsection{7-Triacetylhydrazino-5-aryl-2-phenylpyrazolo[1,5-c]pyrimidines 11a-d}

A suspension of 5-aryl-7-hydrazino-2-phenylpyrazolo[1,5-c]pyrimidines 1a-d (1 mmol) in acetic anhydride $(5 \mathrm{~mL})$ was heated under reflux for $1 \mathrm{~h}$ and the mixture was cooled and poured onto crushed ice. The product that separated out was filtered off, washed with water and then dried. It was crystallized from EtOH to give the title compounds 11a-d as colorlees needles.

7-Triacetylhydrazino-2,5-diphenylpyrazolo[1,5-c]pyrimidine (11a). Yield 83\%, $0.35 \mathrm{~g}, \mathrm{mp} 179-180{ }^{\circ} \mathrm{C}$; IR $\left(v_{\max }, \mathrm{cm}^{-1}\right): 1736(\mathrm{C}=\mathrm{O}), 1623($ pyrazole ring $\mathrm{C}=\mathrm{N}), 1542$ (pyrimidine ring $\left.\mathrm{C}=\mathrm{N}\right)$ and $1458(\mathrm{C}=\mathrm{C})$; ${ }^{1} \mathrm{H}$ NMR (DMSO- $\left.d_{6}, \delta_{\mathrm{H}}, \mathrm{ppm}\right): 2.45\left(\mathrm{~s}, 3 \mathrm{H}, \mathrm{COCH}_{3}\right), 2.48$ (s, 3H, $\left.\mathrm{COCH}_{3}\right), 2.50$ (s, 3H, $\left.\underline{\mathrm{COCH}}_{3}\right), 7.28$ $(\mathrm{s}, 1 \mathrm{H}$, pyrazole-H), $7.44(\mathrm{t}, 2 \mathrm{H}$, aromatic- $\mathrm{H}), 7.50(\mathrm{t}, 4 \mathrm{H}$, aromatic- $\mathrm{H}), 7.99(\mathrm{~d}, 2 \mathrm{H}$, aromatic- $\mathrm{H}), 8.05$ $(\mathrm{d}, 2 \mathrm{H}$, aromatic- $\mathrm{H})$ and $8.24(\mathrm{~s}, 1 \mathrm{H}$, pyrimidine- $\mathrm{H}) ; \mathrm{MS}, m / z(\%)=428\left(\mathrm{M}^{+}+1,15\right), 385$ $\left(\mathrm{M}^{+}-\mathrm{CH}_{2} \mathrm{CO}, 15\right), 343\left(\mathrm{M}^{+}-2 \mathrm{CH}_{2} \mathrm{CO}, 100\right), 301\left(\mathrm{M}^{+}-3 \mathrm{CH}_{2} \mathrm{CO}, 65\right), 286\left(\mathrm{M}^{+}-\mathrm{C}_{6} \mathrm{H}_{6} \mathrm{NO}_{2}, 24\right), 272$ $\left(\mathrm{M}^{+}-\mathrm{C}_{6} \mathrm{H}_{6} \mathrm{~N}_{2} \mathrm{O}_{2}, 88\right)$ and $270\left(\mathrm{M}^{+}-\mathrm{C}_{6} \mathrm{H}_{8} \mathrm{~N}_{2} \mathrm{O}_{2}, 26\right)$; Anal. Calc. for $\mathrm{C}_{24} \mathrm{H}_{21} \mathrm{~N}_{5} \mathrm{O}_{3}$ (427.46): C, 67.44; $\mathrm{H}$, $4.95 ; \mathrm{N}, 16.38 \%$, found: C, 67.40; H, 5.00; N, 16.40\%.

7-Triacetylhydrazino-2-phenyl-5-p-tolylpyrazolo[1,5-c]pyrimidine (11b). Yield 80\%, $0.35 \mathrm{~g}, \mathrm{mp}$ 194-195 ${ }^{\circ} \mathrm{C}$; IR $\left(v_{\max }, \mathrm{cm}^{-1}\right)$ : $1726(\mathrm{C}=\mathrm{O}), 1622$ (pyrazole ring $\left.\mathrm{C}=\mathrm{N}\right), 1520$ (pyrimidine $\operatorname{ring} \mathrm{C}=\mathrm{N}$ ) and $1450(\mathrm{C}=\mathrm{C}) ;{ }^{1} \mathrm{H} \mathrm{NMR}\left(\mathrm{CDCl}_{3}, \delta_{\mathrm{H}}, \mathrm{ppm}\right): 2.17\left(\mathrm{~s}, 3 \mathrm{H}, \mathrm{CH}_{3}\right), 2.42\left(\mathrm{~s}, 3 \mathrm{H}, \mathrm{COCH} \underline{\mathrm{C}}_{3}\right), 2.54\left(\mathrm{~s}, 3 \mathrm{H}, \mathrm{COCH}_{3}\right)$, $2.56\left(\mathrm{~s}, 3 \mathrm{H}, \mathrm{COCH}_{3}\right), 6.90(\mathrm{~s}, 1 \mathrm{H}$, pyrazole- $\mathrm{H}), 7.28(\mathrm{~d}, 2 \mathrm{H}$, aromatic-H), 7.42-7.50 (m, 3H, aromatic$\mathrm{H}), 7.72(\mathrm{~s}, 1 \mathrm{H}$, pyrimidine- $\mathrm{H}), 7.87(\mathrm{~d}, 2 \mathrm{H}$, aromatic- $\mathrm{H})$ and $7.95(\mathrm{~d}, 2 \mathrm{H}$, aromatic- $\mathrm{H}) ; \mathrm{MS}, \mathrm{m} / z(\%)=$ $443\left(\mathrm{M}^{+}+2,2\right), 441\left(\mathrm{M}^{+}, 10\right), 399\left(\mathrm{M}^{+}-\mathrm{COCH}_{2}, 11\right), 357\left(\mathrm{M}^{+}-2 \mathrm{COCH}_{2}, 100\right), 315\left(\mathrm{M}^{+}-3 \mathrm{COCH}_{2}, 44\right)$ and $300\left(\mathrm{M}^{+}-\mathrm{C}_{6} \mathrm{H}_{7} \mathrm{NO}_{3}, 21\right)$; Anal. Calc. for $\mathrm{C}_{25} \mathrm{H}_{23} \mathrm{~N}_{5} \mathrm{O}_{3}$ (441.48): C, 68.01; H, 5.25; N, 15.86\%, found: C, 68.12; H, 5.22; N, 15.81\%.

7-Triacetylhydrazino-5-p-methoxyphenyl-2-phenylpyrazolo[1,5-c]pyrimidine (11c). Yield 76\%, $0.35 \mathrm{~g}$, mp 132-133 ${ }^{\circ} \mathrm{C}$; IR $\left(v_{\max }, \mathrm{cm}^{-1}\right): 1720(\mathrm{C}=\mathrm{O}), 1614($ pyrazole ring $\mathrm{C}=\mathrm{N}), 1510$ (pyrimidine ring $\left.\mathrm{C}=\mathrm{N}\right)$, and $1448(\mathrm{C}=\mathrm{C}) ;{ }^{1} \mathrm{H} \mathrm{NMR}\left(\mathrm{CDCl}_{3}, \delta_{\mathrm{H}}, \mathrm{ppm}\right): 2.53\left(\mathrm{~s}, 9 \mathrm{H}, 3 \mathrm{COCH}_{3}\right), 3.86\left(\mathrm{~s}, 3 \mathrm{H}, \underline{\mathrm{OCH}}_{3}\right), 6.87(\mathrm{~s}, 1 \mathrm{H}$, pyrazole-H), $6.98(\mathrm{~d}, 2 \mathrm{H}$, aromatic- $\mathrm{H}), 7.41-7.49(\mathrm{~m}, 3 \mathrm{H}$, aromatic- $\mathrm{H}), 7.65(\mathrm{~s}, 1 \mathrm{H}$, pyrimidine- $\mathrm{H})$ and 7.91-7.95 (m, 4H, aromatic-H); MS, $m / z(\%)=459\left(\mathrm{M}^{+}+2,2\right), 457\left(\mathrm{M}^{+}, 17\right), 415\left(\mathrm{M}^{+}-\mathrm{COCH}_{2}\right.$, 19), $373\left(\mathrm{M}^{+}-2 \mathrm{COCH}_{2}, \quad 100\right), 331\left(\mathrm{M}^{+}-3 \mathrm{COCH}_{2}, \quad 39\right), 316\left(\mathrm{M}^{+}-\mathrm{C}_{6} \mathrm{H}_{7} \mathrm{NO}_{3}, \quad 17\right)$ and 302 $\left(\mathrm{M}^{+}-\mathrm{C}_{7} \mathrm{H}_{9} \mathrm{NO}_{3}, 41\right)$; Anal. Calc. for $\mathrm{C}_{25} \mathrm{H}_{23} \mathrm{~N}_{5} \mathrm{O}_{4}$ (457.48): C, 65.63; H, 5.07; N, 15.31\%, found: C, $65.51 ; \mathrm{H}, 5.05 ; \mathrm{N}, 15.28 \%$.

7-Triacetylhydrazino-5-p-chlorophenyl-2-phenylpyrazolo[1,5-c]pyrimidine (11d). Yield 76\%, $0.35 \mathrm{~g}$, mp $174-175^{\circ} \mathrm{C}$; IR $\left(v_{\max }, \mathrm{cm}^{-1}\right)$ : $1722(\mathrm{C}=\mathrm{O}), 1616$ (pyrazole ring $\left.\mathrm{C}=\mathrm{N}\right), 1533$ (pyrimidine ring $\left.\mathrm{C}=\mathrm{N}\right)$, and $1443(\mathrm{C}=\mathrm{C}) ;{ }^{1} \mathrm{H} \mathrm{NMR}\left(\mathrm{CDCl}_{3}, \delta_{\mathrm{H}}, \mathrm{ppm}\right): 2.52\left(\mathrm{~s}, 3 \mathrm{H}, \mathrm{COCH}_{3}\right), 2.56\left(\mathrm{~s}, 6 \mathrm{H}, 2 \mathrm{COCH}_{3}\right), 6.91(\mathrm{~s}, 1 \mathrm{H}$, pyrazole-H), 7.41-7.49 (m, 5H, aromatic-H), $7.70(\mathrm{~s}, 1 \mathrm{H}$, pyrimidine- $\mathrm{H}), 7.88(\mathrm{~d}, 2 \mathrm{H}$, aromatic-H) and $7.93\left(\mathrm{~d}, 2 \mathrm{H}\right.$, aromatic-H); MS, $m / z(\%)=464\left(\mathrm{M}^{+}+2,1\right), 462\left(\mathrm{M}^{+}, 5\right), 420\left(\mathrm{M}^{+}-\mathrm{COCH}_{2}, 8\right), 378$ 
$\left(\mathrm{M}^{+}-2 \mathrm{COCH}_{2}, 58\right), 336\left(\mathrm{M}^{+}-3 \mathrm{COCH}_{2}, 30\right), 320\left(\mathrm{M}^{+}-\mathrm{C}_{6} \mathrm{H}_{8} \mathrm{NO}_{3}, 13\right)$ and $306\left(\mathrm{M}^{+}-\mathrm{C}_{6} \mathrm{H}_{8} \mathrm{~N}_{2} \mathrm{O}_{3}, 24\right)$; Anal. Calc. for $\mathrm{C}_{24} \mathrm{H}_{20} \mathrm{ClN}_{5} \mathrm{O}_{3}$ (461.90): C, 62.41; H, 4.36; N, 15.16\%, found: C, 62.40; H, 4.32; N, 15.20\%.

\section{Conclusions}

In summary, the strategy for constructing the target compounds started by 5-aryl-7-hydrazino-2phenylpyrazolo[1,5-c]pyrimidines 1a-d where the hydrazine moiety can be readily heterocyclized with one-carbon inserting agents to give the triazole ring fused to the pyrazolopyrimidine skeleton has been successfully demonstrated.

\section{References}

1. Elnagdi, M.H.; Fleita, D.H.; Elmoghayar, M.R.H. Reactions with $\beta$-cyanoethylhydrazine-II: Synthesis of some 4,5,6,7-tetrahydropyrazolo[1,5-a]pyrimidine derivatives. Tetrahedron 1975, 31, 63-67.

2. Marei, M.G.; Mishrikey, M.M.; Aly, D.M. New Synthesis of Pyrazolo(1,5-c)pyrimidines from Acetylenic $\beta$ - Diketones. Bull. Chem. Soc. Jpn. 1992, 65, 3419-3422.

3. Zvezdina, E.A.; Zhdanova, M.P.I.; Nechayuk, I.I.; Barchan, A.; Simkina, Y.N.; Buchnaya, T.A. Investigation of pharmacological properties of some pyridinium salts, pyrazolines and pyrazoles. Khim. Farm. Zh. 1986, 20, 1328-1331.

4. Marei, M.G.; Hassaan, A.M.A. Nickel(II) and copper(II) complexes of 5-aryl-2-phenyl-6Hpyrazolo-[1,5-c]pyrimidine-7-thiones. Transition Met. Chem. 1992, 17, 489-490.

5. Grim, J.A.; Petering, H.G. The Antitumor Activity of Cu(II)KTS, the Copper(II) chelate of 3ethoxy-2-oxobutyraldehyde bis(thiosemicarbazone). Cancer Res. 1967, 27, 1278-1285.

6. Scovill, J.P.; Klayman, D.L.; Franchino, C.F. 2-Acetylpyridine thiosemicarbazones. 4. Complexes with transition metals as antimalarial and antileukemic agents. J. Med. Chem. 1982, 25, 1261-1264.

7. Sartorelli, A.C.; Creasey, W.A. Cancer chemotherapy. Ann. Rev. Pharmacol. 1969, 9, 51-72.

8. Brucato, A.; Coppola, A.; Gianguzza, S.P.; Provenzano, M. Triazolam: Characteristics of its depressive action. Boll. Soc. Ital. Biol. Sper. 1978, 54, 1051-1057.

9. Coffen, D.L.; Fryer, R.I.; Triazolobenzodiazepines. U.S. Patent 3,849,434, 19 November 1974; [Chem. Abstr. 1975, 82, 73044v].

10. Shiroki, M.; Tahara, T.; Araki, K. Jap. Patent 75100096, 1975; [Chem. Abstr. 1976, 84, 59588k].

11. Povelitsa, F.D.; Gural, A.G. Antibacterial effect of furacriline on Brucella. Antibiotiki 1973, 18, 71-74; [Chem. Abstr. 1973, 78, 93044].

12. Karegoudar, P.; Prasad, D.J.; Ashok, M.; Mahalinga, M.; Poojary, B.; Holla, B.S. Synthesis, antimicrobial and anti-inflammatory activities of some 1,2,4-triazolo[3,4- $b][1,3,4]$ thiadiazoles and 1,2,4-triazolo[3,4-b][1,3,4]thiadiazines bearing trichlorophenyl moiety. Eur. J. Med. Chem. 2008, 43, 808-815.

13. Heindel, N.D.; Reid, J.R. 4-Amino-3-mercapto-4H-1,2,4-triazoles and propargyl aldehydes: A new route to 3-R-8-aryl-1,2,4-triazolo[3,4-b]-1,3,4-thiadiazepines. J. Heterocycl. Chem. 1980, 17, 1087-1088. 
14. Holla, B.S.; Kalluraya, B.; Sridhar, K.R.; Drake, E.; Thomas, L.M.; Bhandary, K.K.; Levine, M.S. Synthesis, structural characterization, crystallographic analysis and antibacterial properties of some nitrofuryl triazolo[3,4-b]-1,3,4-thiadiazine. Eur. J. Med. Chem. 1994, 29, 301-308.

15. Mathew, V.; Keshavayya, J.; Vidya, V.P.; Acharya; Reddy, B.M. Heterocyclic system containing bridgehead nitrogen atom: Synthesis and pharmacological activities of some substituted 1,2,4triazolo[3,4-b]-1,3,4-thiadiazoles. Eur. J. Med. Chem. 2006, 41, 1048-1058.

16. Budavari, S.; O'Neil, M.; Smith, A.; Heckelman, P.; Obenchain, J. The Merck Index an Encyclopedia of Chemicals, Drugs and Biologicals, 12th ed.; Merck Co. Inc.: Rahway, NJ, USA, 1996.

17. Haber, J. Present status and perspectives on antimycoties with systematic effects. Cas. Lek. Cesk. 2001, 140, 596.

18. Atta, K.F.; El Massry, A.M.; Abdel Hamid, H.; El Ashry, E.S.H.; Amer, A. Synthesis of 4(Pyrazol-3-yl)[1,2,4]triazolo[4,3-a]quinoxalines and tetrazolo analog [I]. J. Heterocycl. Chem. 1994, 31, 549-552.

19. Atta, K.F.M.; Marei, M.G.; Mohamed, F.A.M. Synthesis and reactions of a new series of 1,2,4-triazolo[4,3-c]quinazolines. Heterocycles 2011, 83, 339-349.

20. Atta, K.F.M.; Marei, M.G.; Abd El-Magiad, S.M.; El-Nashar, F.H.A. Annulation and evaluation of antibacterial activity of the new fused tricyclic $(5,5,6)$ ring system of pyrazolo[1,5-c]1,2,4-triazolo[4,3-a]pyrimidines. Heterocycles 2011, 83, 1873-1888.

21. Atta, K.F.M.; El Ashry, E.S.H. Synthesis of 4-(1-phenyl-1H-pyrazol-3-yl)[1,2,4]triazolo[4,3-a] quinoxalines and their 4-halogenopyrazolyl analogs. J. Heterocycl. Chem. 2011, in press.

22. Marei, M.G.; El-Ghanam, M. Reaction of pyrazolo[1,5-c]pyrimidinethiones with hydrazine: Synthesis of novel substituted pyrazolo[1,5-c]pyrimidine and pyrazolo[1,5-c]tetrazolo[1,5a]pyrimidine derivatives. J. Chem. Res. 1993, 2175-2188.

Sample Availability: Samples of compounds 1-11 are available from the author.

(C) 2011 by the authors; licensee MDPI, Basel, Switzerland. This article is an open access article distributed under the terms and conditions of the Creative Commons Attribution license (http://creativecommons.org/licenses/by/3.0/). 